\title{
Characterization and history of the Helmi streams with Gaia DR2
}

\author{
Helmer H. Koppelman ${ }^{1}$, Amina Helmi ${ }^{1}$, Davide Massari ${ }^{1}$, Sebastian Roelenga ${ }^{1}$, and Ulrich Bastian ${ }^{2}$ \\ 1 Kapteyn Astronomical Institute, University of Groningen, Landleven 12, 9747 AD Groningen, The Netherlands \\ e-mail: koppelman@astro.rug.nl \\ 2 Zentrum für Astronomie, Heidelberg University, Astronomisches Rechen-Institut, Mönchhofstrasse 12-14, \\ 69120 Heidelberg, Germany
}

Received 3 December 2018 / Accepted 20 March 2019

\begin{abstract}
Context. The halo of the Milky Way has long been hypothesized to harbour significant amounts of merger debris. For more than a decade this view has been supported by wide-field photometric surveys which have revealed the outer halo to be lumpy.

Aims. The recent release of Gaia DR2 is allowing us to establish that mergers also have been important and possibly built up the majority of the inner halo. In this work we focus on the Helmi streams, a group of streams crossing the solar vicinity and known for almost two decades. We characterize their properties and relevance for the build-up of the Milky Way's halo.

Methods. We identify new members of the Helmi streams in an unprecedented dataset with full phase-space information combining Gaia DR2, and the APOGEE DR2, RAVE DR5, and LAMOST DR4 spectroscopic surveys. Based on the orbital properties of the stars, we find new stream members up to a distance of $5 \mathrm{kpc}$ from the Sun, which we characterized using photometry and metallicity information. We also perform N-body experiments to constrain the time of accretion and properties of the progenitor of the streams. Results. We find nearly 600 new members of the Helmi streams. Their HR diagram reveals a broad age range, from $\approx 11$ to $13 \mathrm{Gyr}$, while their metallicity distribution goes from -2.3 to -1.0 , and peaks at $[\mathrm{Fe} / \mathrm{H}] \sim-1.5$. These findings confirm that the streams originate in a dwarf galaxy. Furthermore, we find seven globular clusters to be likely associated, and which follow a well-defined age-metallicity sequence whose properties suggest a relatively massive progenitor object. Our N-body simulations favour a system with a stellar mass of $\sim 10^{8} M_{\odot}$ accreted 5-8 Gyr ago.

Conclusions. The debris from the Helmi streams is an important donor to the Milky Way halo, contributing $\approx 15 \%$ of its mass in field stars and $10 \%$ of its globular clusters.
\end{abstract}

Key words. Galaxy: halo - Galaxy: kinematics and dynamics - solar neighborhood

\section{Introduction}

According to the concordance cosmological model $\Lambda \mathrm{CDM}$, galaxies grow by mass through mergers. Typically a galaxy's halo is formed through a handful of major mergers accompanied by a plethora of minor mergers. This model's predictions stem both from dark-matter-only simulations (Helmi et al. 2003) combined with semi-analytic models of galaxy formation (e.g., Bullock \& Johnston 2005; Cooper et al. 2010) and hydrodynamical simulations (e.g., Pillepich et al. 2014).

When satellites merge with a galaxy like the Milky Way they get stripped of their stars by the tidal forces (e.g., Johnston et al. 1996). These stars follow approximately the mean orbit of their progenitor and this leads to the formation of streams and shells. Wide-field photometric surveys have already discovered many cold streams likely due to globular clusters, for example Pal 5 (Odenkirchen et al. 2001); GD-1 (Grillmair \& Dionatos 2006), and more disperse streams caused by dwarf galaxies, for example Sagittarius (Ibata et al. 1994), whose total extent and importance can only be appreciated in full-sky maps (Belokurov et al. 2006; Bernard et al. 2016; Shipp et al. 2018). Many of these streams are distant and have become apparent only after meticulous filtering (e.g., Rockosi et al. 2002; Grillmair 2009; Malhan et al. 2017).

Tidal debris in the vicinity of the Sun is predicted to be very phase-mixed (Helmi \& White 1999; Helmi et al. 2003). Typically one can expect to find many stream wraps originating in the same object, that is groups of stars with different orbital phase sharing a common origin. Rather than to search for substructure in spatial coordinates, it is more productive to study tidal debris in spaces where the degree of clustering is either constant, such as in integrals of motion or action space (Helmi \& de Zeeuw 2000; Mcmillan \& Binney 2008), or even increases with time (Helmi \& White 1999). Until recently, a few studies on the nearby stellar halo identified different small groups of stars that likely were accreted together (Helmi et al. 1999, 2006, 2017; Chiba \& Beers 2000; Klement et al. 2008, 2009; Williams et al. 2011; Majewski et al. 2012; Beers et al. 2017), see Newberg \& Carlin (2016) for a comprehensive review.

A new era is dawning now that the Gaia mission is delivering full phase-space information for a billion stars. As it is clear from the above discussion, this is crucial to unravel the merger history of the Milky Way and to characterize the properties of the satellites with which it merged. The strength of Gaia, and especially of the $6 \mathrm{D}$ sample, is that it can identify stream members based on the measured kinematics (e.g., Koppelman et al. 2018; Price-Whelan \& Bonaca 2018). In fact, the second data release of the Gaia mission (Gaia Collaboration 2018b) is already transforming the field of galactic archaeology. One example is the recent spectacular discovery that the inner halo was built largely via the accretion of a single object, as first hinted from the kinematics (Belokurov et al. 2018; Koppelman et al. 2018), and the stellar populations (Gaia Collaboration 2018a; Haywood et al. 2018), all pieces put together in Helmi et al. (2018). This accreted 
system, known as Gaia-Enceladus, was discy and similar in mass to the Small Magellanic Cloud of today, and hence led to the heating of the galactic proto-disc some 10 Gyr ago (Helmi et al. 2018).

Gaia-Enceladus debris, however, is not the only substructure present in the vicinity of the Sun. Detected about 20 years ago, the Helmi streams (Helmi et al. 1999, H99 hereafter) are known to cross the solar neighbourhood. Their existence has been confirmed by Chiba \& Beers (2000) and Smith et al. (2009), among other studies. In the original work, 13 stars were detected based on their clumped angular momenta which clearly differ from other local halo stars. Follow-up work by Kepley et al. (2007) estimated that the streams were part of the tidal debris of a dwarf galaxy that was accreted 6-9 Gyr ago, based on the bimodality of the $z$-velocity distribution. This bimodal distribution is the distinctive feature of multiple wraps of tidal debris crossing the solar neighbourhood. Since the discovery in 1999, a handful of new tentative members have been found, increasing the total number of members to $~ 30$ (e.g., Kepley et al. 2007; Re Fiorentin et al. 2005; Klement et al. 2009; Beers et al. 2017), while several tens more were reported in Gaia Collaboration (2018c). Also structure S2 from Myeong et al. (2018a), consisting of $\sim 60$ stars, has been recognized to be related to the Helmi streams (W. Evans priv. comm.).

Originally, the Helmi streams were found using Hipparcos proper motions (Perryman et al. 1997) combined with groundbased radial velocities (Beers \& Sommer-Larsen 1995; Chiba \& Yoshii 1998). In this work, we aim to find new members and to characterize better its progenitor in terms of the time of accretion, initial mass and star formation history. To this end, we focus on the dynamics, metallicity distribution and colour-magnitude diagram of its members. Furthermore, we also identify globular clusters that could have potentially been accreted with the object (Leaman et al. 2013; Kruijssen et al. 2018), as for example seen for the Sagittarius dwarf galaxy (Law \& Majewski 2010; Massari et al. 2017; Sohn et al. 2018), and also for GaiaEnceladus (Myeong et al. 2018b; Helmi et al. 2018).

This paper is structured as follows: in Sect. 2 we present the data and samples used, while in Sect. 3 we define a core selection of streams members that serves as the basis to identify more members. In Sect. 4 we analyse the spatial distribution of the debris. In Sect. 5 we supplement the observations with $\mathrm{N}$-body simulations. We discuss possible associations of the Helmi streams with globular clusters in Sect. 6. Finally, we present our conclusions in Sect. 7.

\section{Data}

\subsection{Brief description of the data}

The recently published second data release (DR2) of the Gaia space mission contains the on-sky positions, parallaxes, proper motions, and the $G, G_{\mathrm{BP}}$ and $G_{\mathrm{RP}}$ optical magnitudes for over 1.3 billion stellar sources in the Milky Way (Gaia Collaboration 2018b). For 7224631 stars with $G_{\mathrm{RVs}}<12$, known as the 6D subsample, line-of-sight velocity information measured by the Gaia satellite is available (Gaia Collaboration 2018d). The precision of the observables in this dataset is unprecedented: the median proper motions uncertainties of the stars with full phasespace information is $1.5 \mathrm{mas} / \mathrm{yr}$ which translates to a tangential velocity error of $\sim 7 \mathrm{~km} \mathrm{~s}^{-1}$ for a star at $1 \mathrm{kpc}$, while their median radial velocity uncertainties are $3.3 \mathrm{~km} \mathrm{~s}^{-1}$. This makes the Gaia DR2 both the highest-quality and the largest-size single survey ever available for studying the kinematics and dynamics of the nearby stellar halo and disc.

\subsection{Cross-matching with APOGEE, RAVE, and LAMOST}

To supplement the 6D Gaia subsample, we add the radial velocities from the cross-matched catalogues APOGEE (Wilson et al. 2010; Abolfathi et al. 2018) and RAVE DR5 (Kunder et al. 2017), see Marrese et al. (2018) for details. We also add radial velocities from our own cross-match of Gaia DR2 with LAMOST DR4 (Cui et al. 2012).

For the cross-match with LAMOST we first transform the stars to the same reference frame using the Gaia positions and proper motions, and then we match stars within a radius of 10 arcsec with TOPCAT/STILTS (Taylor 2005, 2006). We find that over $95 \%$ of the stars have a matching radius smaller than 0.5 arcsec. In total, we find 2868425 matches between Gaia and LAMOST DR4, with a subset of 8404 overlapping also with RAVE, and 50650 with APOGEE. Because the LAMOST radial velocities are known to be offset by $+4.5 \mathrm{~km} \mathrm{~s}^{-1}$ with respect to APOGEE (Anguiano et al. 2018), we correct for this effect.

Since the radial velocities of RAVE and APOGEE have been shown to be very consistent with those of Gaia (Sartoretti et al. 2018), for our final catalogue we use first the radial velocities from APOGEE if available, then those from RAVE, and finally from LAMOST for the stars for which there is no overlap with either two of the other surveys. After imposing a quality cut of parallax_over_error $>5$, this yields a sample of 2361519 stars with radial velocities. We note that all these surveys also provide additional metallicity information for a subset of the stars.

When combined with the Gaia 6D sample, this results in a total of 8738322 stars with 6D information and parallax_over_error $>5$. The median line-of-sight velocity error of the stars from the ground-based spectroscopic surveys is $5.8 \mathrm{~km} \mathrm{~s}^{-1}$, while that of the pure Gaia sample is $1 \mathrm{~km} \mathrm{~s}^{-1}$ for the same parallax quality cut.

\subsection{Quality cuts and halo selection}

To isolate halo stars, we follow a kinematic selection, meaning that stars are selected on the basis of their very different velocity from local disc stars. By cutting in velocity we introduce a clear bias: halo stars with disc-like kinematics are excluded from this sample (Nissen \& Schuster 2010; Bonaca et al. 2017; Posti et al. 2018; Koppelman et al. 2018). Nevertheless, the amplitude of the Z-velocities of the Helmi streams stars is $>200 \mathrm{~km} \mathrm{~s}^{-1}$, that is very different from the disc, so our selection will not impact our ability to find more members. Such a selection reduces significantly the sample size (since by far most stars are in the thin disc) and helps in making the Helmi streams more apparent.

We start from our extended 6D data sample (obtained as described in the previous section). Because of the zeropoint offset of $\sim-0.03$ mas known to affect the Gaia parallaxes (Arenou et al. 2018; Gaia Collaboration 2018b; Lindegren et al. 2018) we also discard stars with parallaxes $<0.2$ mas. Distances for this reduced sample are obtained by inverting the parallaxes. Following Koppelman et al. (2018) we select stars that have $\left|\boldsymbol{V}-\boldsymbol{V}_{\mathrm{LSR}}\right|>210 \mathrm{~km} \mathrm{~s}^{-1}$, where $\boldsymbol{V}_{\mathrm{LSR}}$ is the velocity vector of the local standard of rest (LSR). A selection like this removes all thin-disc stars, assuming those move at the LSR velocity. Thick disc stars moving at $\sim 170 \mathrm{~km} \mathrm{~s}^{-1}$ (Morrison et al. 1990), will be mostly removed except for those that rotate slowly and/or have an exceptionally large vertical velocity. We apply the velocity selection after correcting for the motion of the Sun using the values, $\left(U_{\odot}, V_{\odot}, W_{\odot}\right)=(11.1,12.24,7.25) \mathrm{km} \mathrm{s}^{-1}$ 

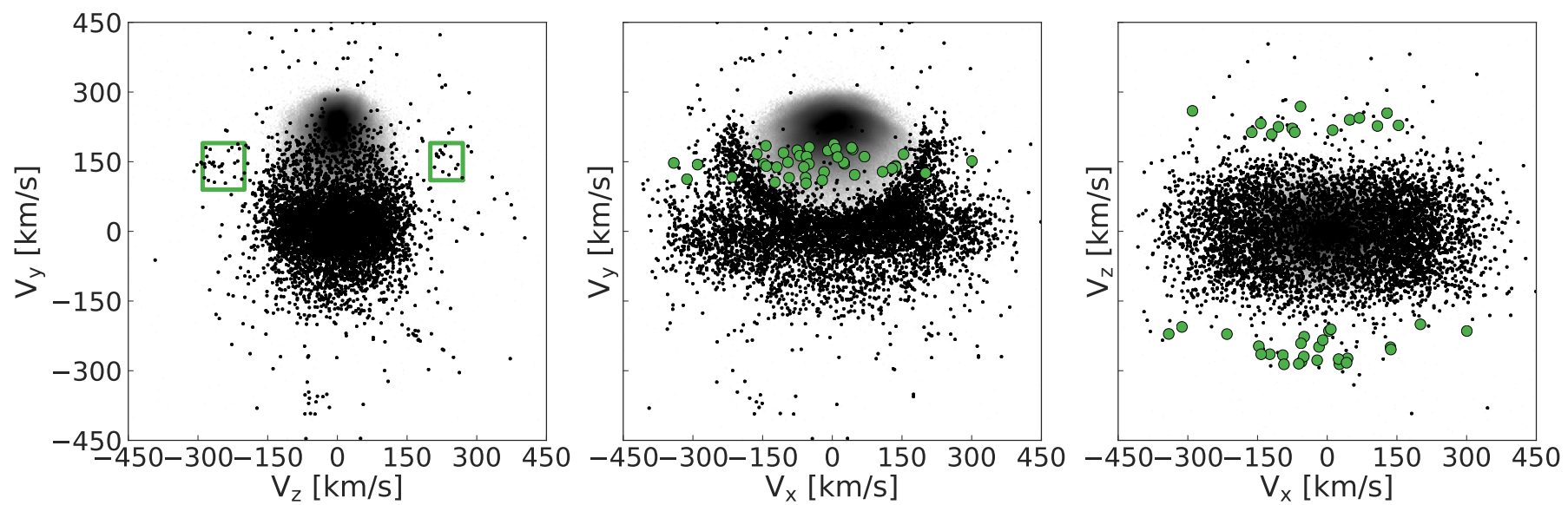

Fig. 1. Velocity distribution of kinematically selected halo stars (black dots) within 1 kpc from the Sun from the Gaia-only 6D sample. The grey density in the background shows the location and extent of the disc in this diagram. The velocities have been corrected for the motion of the Sun and LSR. The green boxes in the left panel indicate the location of the Helmi streams, and are drawn based on the velocities of the original stream members. The stars inside these boxes are highlighted with green symbols in the other two panels.

(Schönrich et al. 2010) and that of the LSR, $V_{\mathrm{LSR}}=232.8 \mathrm{~km} \mathrm{~s}^{-1}$ (Mcmillan 2017). We do not take the uncertainties of the velocities into account, as these are typically very small with a median value of $\sim 4 \mathrm{~km} \mathrm{~s}^{-1}$.

Our Cartesian reference frame is pointed such that $X$ is positive towards the Galactic centre, $Y$ points in the direction of the motion of the disc, and $Z$ is positive for Galactic latitude $b>0$. In this frame the Sun is located at $X=-8.2 \mathrm{kpc}$ from the Galactic centre (Mcmillan 2017). This final sample contains 79318 tentative halo stars, with 12472 located within $1 \mathrm{kpc}$ from the Sun. Slightly more than half of these stars stem from the Gaiaonly $6 \mathrm{D}$ sample.

\section{Finding members}

\subsection{Core selection}

Using the halo sample described above, we select "core members" by considering only those stars within $1 \mathrm{kpc}$ from the Sun from the Gaia-only sample. The parallaxes for this subsample are very good, with a median parallax_over_error of 46 . In such a local sample, streams are very clustered in velocityspace because the gradients caused by the orbital motion are minimized. In the next section we will use the orbits of these core members to find members at distances beyond $1 \mathrm{kpc}$.

Figure 1 shows our selection of the streams' core members in velocity space with green boxes. The boxes are placed on top of the positions of the original H99 members of the Helmi streams. The boundaries in $\left(V_{Z}, V_{Y}\right)$ for the left box are: $[-290,-200][90,190] \mathrm{km} \mathrm{s}^{-1}$, and for the right box: $[200,270][110,190] \mathrm{km} \mathrm{s}^{-1}$. Using the SIMBAD database we find that ten of the original 13 members have Gaia DR2 distances smaller than $1 \mathrm{kpc}$. Of these ten stars, nine have radial velocities in our extended data sample. Only one star with updated radial velocity information has very different velocities, leaving the original sample with eight reliable members with full Gaia 6D parameters within $1 \mathrm{kpc}$.

One of the key characteristics of the Helmi streams are the two groups in the $V_{Y}-V_{Z}$ plane, one moving with positive $V_{Z}$ and one with negative $V_{Z}$. Using the selection described above we find 40 core members in the Gaia-only sample, of which 26 with $V_{Z}<0$. The Gaia DR2 source_ids are given in Table 1. While we use the kinematically biased halo sample to find these
Table 1. Gaia DR2 source_id of the Helmi streams' core members.

\begin{tabular}{cc}
\hline \hline IDs members $1-20$ & IDs members $21-40$ \\
\hline 365903386527108864 & 604095572614068352 \\
640225833940128256 & 1049376272667191552 \\
1415635209471360256 & 1621470761217916800 \\
1639946061258413312 & 2075971480449027840 \\
2081319509311902336 & 2268048503896398720 \\
2322233192826733184 & 2416023871138662784 \\
2447968154259005952 & 2556488440091507584 \\
2604228169817599104 & 2670534149811033088 \\
2685833132557398656 & 2891152566675457280 \\
3085891537839264896 & 3085891537839267328 \\
3202308378739431936 & 3214420461393486208 \\
3306026508883214080 & 3742101345970116224 \\
4440446153372208640 & 4768015406298936960 \\
4998741805354135552 & 5032050552340352384 \\
5049085217270417152 & 5388612346343578112 \\
5558256888748624256 & 5986385619765488384 \\
6050982889930146304 & 6170808423037019904 \\
6221846137890957312 & 6322846447087671680 \\
6336613092877645440 & 6545771884159036928 \\
6615661065172699776 & 6914409197757803008 \\
\hline
\end{tabular}

core members, there are no stars that did not make it to the core sample because of this. The halo sample is used here to illustrate how conspicuous the streams are with respect to the background halo in velocity space. The asymmetry in the number of stars in the two groups can be used as an indicator of the time of accretion and/or mass of the progenitor since tidal streams will only produce multiple wraps locally after having evolved for a sufficiently long time. In Sect. 5.1 we explore what the observed asymmetry implies for the properties of the progenitor of the Helmi streams.

\subsection{Beyond the core selection}

The original $13 \mathrm{H} 99$ members are located within $2.5 \mathrm{kpc}$ from the Sun. Beyond this distance, we expect that other members of the streams will have different kinematic properties because 
of velocity gradients along their orbit. Therefore the best way of finding more members beyond the local volume is to use integrals of motion (IOM) such as angular momenta and energy or the action integrals (Helmi \& de Zeeuw 2000). From this section onward, we use the extended sample described in Sect. 2.2 and which includes radial velocities from APOGEE/ RAVE/LAMOST.

We will mainly base the membership selection on the angular momentum of the stars, namely the $z$-component $L_{z}$, and the perpendicular component: $L_{\perp}=\sqrt{L_{x}^{2}+L_{y}^{2}}$, although the latter is not fully conserved in general. Since the energy $E$ depends on the assumed model for the galactic potential, we use it only to check for outliers.

To calculate the energy of the stars we model the Milky Way with a potential that is similar to that used by Helmi et al. (2017): it includes a Miyamoto-Nagai disc with parameters $M_{\mathrm{d}}=9.3 \times 10^{10} M_{\odot},\left(a_{\mathrm{d}}, b_{\mathrm{d}}\right)=(6.5,0.26) \mathrm{kpc}$, an NFW halo with parameters $M_{\mathrm{h}}=10^{12} M_{\odot}, r_{\mathrm{s}, \mathrm{h}}=21.5 \mathrm{kpc}, c_{\mathrm{h}}=12$, and a Hernquist bulge with parameters $M_{\mathrm{b}}=3 \times 10^{10} M_{\odot}, c_{\mathrm{b}}=0.7 \mathrm{kpc}$. The circular velocity curve of this potential is similar to that of the Milky Way. Since this potential is axisymmetric $L_{z}$ is a true IOM. For convenience, in what follows we flip the sign of $L_{z}$ such that it is positive for the Sun.

In Fig. 2 we show the distribution of $L_{z}$ versus $L_{\perp}$ (top), and $L_{z}$ versus $E$ (bottom). A grey density map of all the stars in the Gaia 6D sample with $20 \%$ relative parallax error and with parallax $>0.2$ marks the location of the disc. The black dots correspond to all kinematically selected halo stars within $2.5 \mathrm{kpc}$ from the Sun. The transition of the halo into the disc is smooth, and only appears sharp because of this particular visualization. The streams are clumped around $\left(L_{\perp}, L_{z}\right) \sim$ $(2000,1250) \mathrm{kpc} \mathrm{km} \mathrm{s}^{-1}$, and are highlighted by overlaying the core members from Sect. 3.1 with green dots. In the bottom panel, we see that some of the core members appear to be outliers with too high or low energy (but the analyses carried out in the next sections show they are indistinguishable in their other properties, except for their large $v_{\mathrm{R}}$ velocities, see Sect. 5.1)

Red, dashed lines indicate two boxes, labelled $A \& B$, that we use to select tentative additional stream members. The limits of box A are: $1750<L_{\perp}<2600 \mathrm{kpc} \mathrm{km} \mathrm{s}^{-1}$ and $1000<$ $L_{z}<1500 \mathrm{kpc} \mathrm{km} \mathrm{s}^{-1}$, and those of box B are: $1600<L_{\perp}<$ $3200 \mathrm{kpc} \mathrm{km} \mathrm{s}^{-1}$ and $750<L_{z}<1700 \mathrm{kpc} \mathrm{km} \mathrm{s}^{-1}$. The boxes are placed on top of the core members, their sizes are chosen somewhat arbitrarily. The selection is tight where we expect to find a large amount of contamination, for example on the lower boundary (towards the disc), and looser for the upper boundary. The exact footprint of the entire stream in this diagram could even be larger than box B depending on the size of the progenitor galaxy (as for example can be seen from the numerical simulations shown in Fig. 15). Our decision to explore the two boxes A\&B allows us to check how the level of contamination changes with box size ${ }^{1}$.

The number of stars located within $5 \mathrm{kpc}$ that fall in boxes $\mathrm{A} \& \mathrm{~B}$ are 235 and 523, respectively. At most 20 stars that we identify as members of the Helmi streams in the IOM space (grey points inside the selection boxes), do not satisfy our halo selection (meaning that they have $\left|\boldsymbol{V}-\boldsymbol{V}_{\mathrm{LSR}}\right|<210 \mathrm{~km} \mathrm{~s}^{-1}$ ). In the following sections, we focus on the streams members that fall in selection $B$ unless mentioned otherwise. We remind the reader that selection $B$ includes stars from the full

\footnotetext{
1 We have explored an even larger box size but noticed that the contamination increased significantly when inspecting for example, the metallicity distribution presented in Sect. 4.4.
}

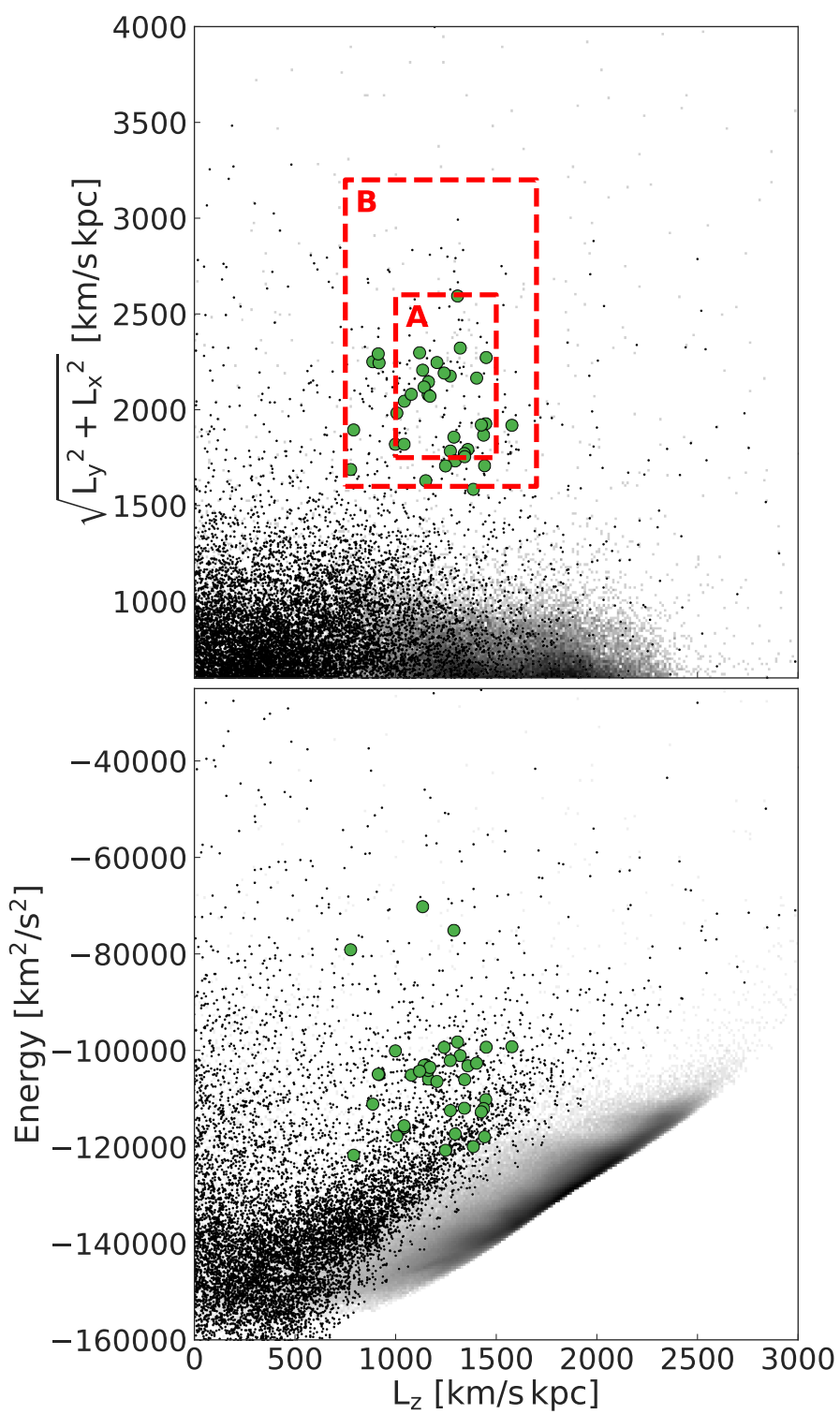

Fig. 2. Distribution of angular momentum $L_{\perp}$ and $L_{z}$ (top panel) energy $E$ and $L_{z}$ (bottom panel). The black dots show all of the stars of our sample of kinematically selected halo stars located within $2.5 \mathrm{kpc}$ from the Sun. Core members from Sect. 3.1 of the Helmi streams are shown with green symbols to guide the reader's eyes. With red, dashed lines we indicate the limits we apply to select additional members of the Helmi streams. In the background, the location of the disc(s) is shown with a grey density map.

extended sample comprising radial velocities from Gaia and from APOGEE/RAVE/LAMOST. All sources in selection B are listed in Table B.1, see Appendix B.

\subsection{Members without radial velocities}

Most of the stars in the Gaia DR2 dataset lack radial velocities, which makes the search for additional tentative members of the Helmi streams less straightforward. In the work of Gaia Collaboration (2018c) new members were identified using locations on the sky where the radial velocity does not enter in the equations for the angular momentum, namely towards the Galactic centre and anti-centre. At those locations, the radial velocity is aligned with the cylindrical $v_{\mathrm{R}}$ component, therefore, it does not contribute to $L_{z}=r v_{\phi}$, and $L_{y}=-x v_{z}$. The degree 
to which the radial velocity contributes to the angular momenta increases with angular distance from these two locations on the sky. Based on a simulation of a halo formed through mergers created by Helmi \& de Zeeuw (2000), we estimate that within $15^{\circ}$ from the (anti-)centre, the maximum difference between the true angular momenta of stars and that computed assuming a zero radial velocity, is $\sim 1000 \mathrm{kpc} \mathrm{km} \mathrm{s}^{-1}$. Since the size of Box $\mathrm{B}$ is $\sim 1000 \mathrm{kpc} \mathrm{km} \mathrm{s}^{-1}$, we consider $15^{\circ}$ as the maximum tolerable search radius. We denote the angular momenta computed assuming zero radial velocity as $\widetilde{L}_{y}$ and $\widetilde{L}_{z}$, where we change the sign of $\widetilde{L}_{z}$ such that it is positive in the (prograde) direction of rotation of the disc.

Therefore, using the full Gaia DR2 5D-dataset, we select stars within $15^{\circ}$ from the (anti-)centre and with parallax over_error $>5$, and apply the following photometric quality cuts described in Sect. 2.1 of Gaia Collaboration (2018a):

phot_g_mean_flux_over_error $>50$,

phot_rp_mean_flux_over_error $>20$,

phot_bp_mean_flux_over_error $>20$,

$1.0+0.015 \times($ bp_rp, 2$)<$ phot_bp_rp_excess_factor,

$1.3+0.06 \times($ bp_rp, 2$)<$ phot_bp_rp_excess_factor.

Figure 3 shows the distribution of all the stars in this subsample with a grey density map in $\widetilde{L}_{y}$ vs $\widetilde{L}_{z}$ space. The two boxes marked with red dashed lines show the criteria we apply to identify additional members of the Helmi streams. The size and location of the boxes are based on those in Fig. 2. They are limited by 750 $<\widetilde{L}_{z}<1700 \mathrm{kpckm} \mathrm{s}^{-1}$, while we use a tighter constraint on $\left|\widetilde{L}_{y}\right|$ to prevent contamination from the disc. The black dashed lines in Fig. 3 indicate upper and lower quantiles of the full $\widetilde{L}_{y^{-}}$ distribution such that $95 \%$ of the stars in the 5D subsample are located between these dashed lines. The lower limits of selection boxes in the $\widetilde{L}_{y}$ direction are offset by $500 \mathrm{kpc} \mathrm{km} \mathrm{s}^{-1}$ from the dashed lines, and are located at $\widetilde{L}_{y}$ at 1782 and $-1613 \mathrm{kpc} \mathrm{km} \mathrm{s}^{-1}$, respectively.

The blue symbols in Fig. 3 correspond to the 105 tentative members that fall inside the boxes. Most of these stars are within $2.5 \mathrm{kpc}$ from the Sun. The two clumps in $\widetilde{L}_{y}$ have a direct correspondence to the two streams seen in the $V_{Z}$ component for the $6 \mathrm{D}$ sample. The clumps have 24 and 81 stars each, implying a 1:3 asymmetry which is quite different from that seen in the number of core member stars associated with each of the two velocity streams. The difference could be caused in part by incompleteness and crowding effects together with an anisotropic distribution of the stars in the streams (see e.g., Fig. 7). We use the 5D members in this work only for the photometric analysis of the Helmi streams carried out in Sect. 4.4.

\section{Analysis of the streams}

\subsection{Spatial distribution}

Figure 4 shows the distribution of the streams members in the $X Z$-plane (left) and $X Y$-plane (right), for the selection box A (top) and for B (bottom). Those identified with $6 \mathrm{D}$ information are indicated with green circles, a local sample of halo stars is shown in grey in the background. There is a lack of stars close to the plane of the disc, likely due to extinction (Gaia Collaboration 2018d). This gap is filled with tentative members from the 5D sample (in blue) which have, by construction, low galactic latitude. Figure 4 reveals the streams stars to be extended along the $Z$-axis, as perhaps expected from their high $V_{Z}$ velocities, but there is also a clear decrease in the number of members with distance from the Sun.

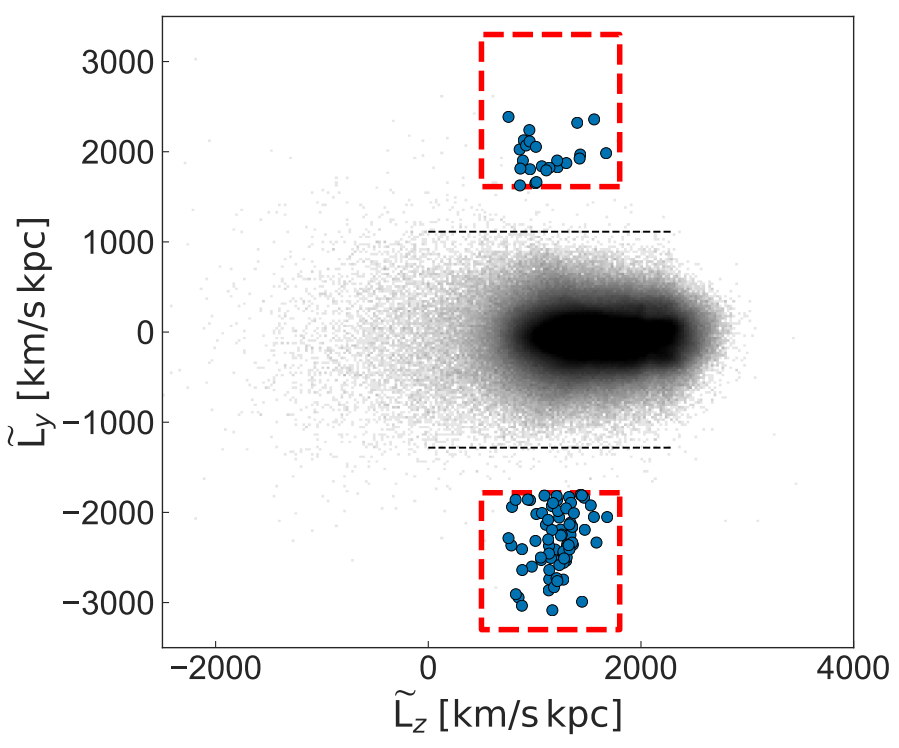

Fig. 3. Distribution of stars from the 5D subset of Gaia, located within $15^{\circ}$ from the Galactic centre or anti-centre, in (pseudo)angular momentum space. The angular momenta are calculated here by assuming that the line-of-sight velocities are zero. The grey density map reveals the location of all of the stars in these windows, most of which are disc stars. The black dashed lines show the $2.5 \%$ and $97.5 \%$ quantiles of the $\widetilde{L}_{y}$-distribution. The two boxes indicated with red dashed lines are used to identify candidate members of the Helmi streams, here shown with blue symbols.

To establish whether the spatial distribution of the streams differs from that of the background, we proceed as follows. We compare our sample of streams stars to $10^{4}$ samples randomly drawn from the background. The random samples contain the same number of stars as the streams, and the background comprises all of the stars in the halo sample, described in Sect. 2, excluding the streams members. In this way, we account for selection effects associated with the different footprints of the APOGEE/RAVE/LAMOST surveys as well as with the $20 \%$ relative parallax error cut (since the astrometric quality of the Gaia data is not uniform across the sky), and which are likely the same for the streams and the background.

Figure 5 shows a comparison of the distribution of heliocentric $R$ (left) and $Z$ (right) coordinates of the stars in our sample (in green) and in the random samples (black). The sizes of the grey and black markers indicate the $1 \sigma$ and $3 \sigma$ levels respectively, of the random samples. The $Z$-distribution of the streams members shows minimal differences with respect to the background, except near the plane $Z \sim 0$. On the other hand, their distribution in $R$ shows very significant differences with respect to the background, depicting a very steeply declining distribution. This was already hinted at in Fig. 6 , and would suggest that the streams near the Sun have a cross section ${ }^{2}$ of $\sim 500 \mathrm{pc}$.

\subsection{Flows: velocity and spatial structure}

The main characteristic of stars in streams is that they move together through space as in a flow. Figure 6 illustrates this by showing the spatial distribution of the members (according to selection B), in the $X Z$-plane. The arrows indicate the direction and amplitude of the velocities of the stars, with stars with

2 Defined as the distance at which the counts of stars has dropped by a factor two. 

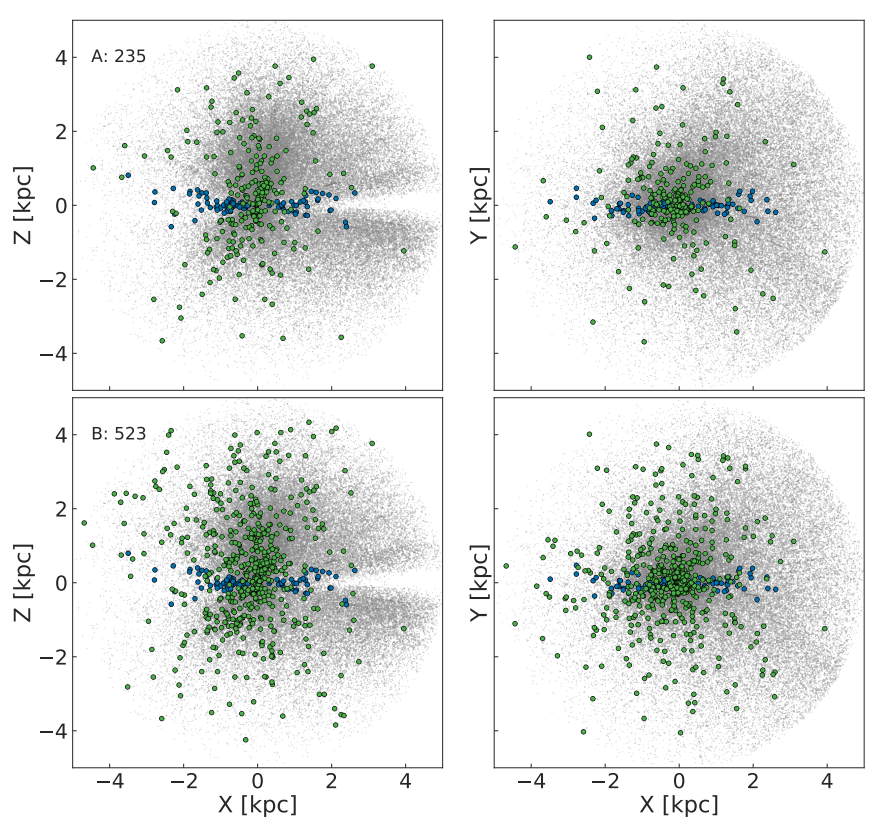

Fig. 4. Spatial distribution of members of the Helmi streams for the two selection boxes A (top) and B (bottom). The 6D stream members are indicated with green circles, local halo stars are shown in the background with grey symbols. The total number of 6D stream members is indicated in the top left of each panel. Tentative members from the 5D dataset are shown with blue symbols.

$V_{Z}<0$ shown in the top row and those with $V_{Z}>0$ in the bottom row of the figure. Every star is colour-coded according to its velocity component in and out of the plane of projection (i.e. its $V_{Y}$ ). The left column shows stars with $V_{X}<0$, while the right column shows stars with $V_{X}>0$.

The flows seen in Fig. 6 reveal that the two characteristic clumps in $V_{Z}$ (i.e. those shown in the left panel of Fig. 1) actually consist of several smaller streams. For example, the top and bottom right panels of Fig. 6 both clearly show two flows: one with $V_{X} \sim 0$, the other with a large $V_{X}$.

To enhance the visibility of the flows we integrate the orbits of the streams stars forward and backwards in time. The potential in which the orbits are calculated is the same as the one described in Sect. 3.2. The trajectories of all the stars are integrated for $\pm 100 \mathrm{Myr}$ in time and are shown in Fig. 7 projected onto the $X Z$ plane. With a red star we indicate the solar position. The trajectories of stars that belong to the group with $V_{Z}>0$ are coloured black, while those with $V_{Z}<0$ are given in blue.

Clearly, the stars found in the solar vicinity are close to an orbital turning point and on trajectories elongated in the $Z$-direction, as expected from their large vertical velocities. Figure 7 serves to understand the observed spatial distribution of the member stars (i.e. narrower in $X$ (or $R$ ) and elongated in $Z$ ) seen in Fig. 4. Finally, we also note the presence of groups of orbits tracing the different flows just discussed, such as for example the group of stars moving towards the upper left corner of the figure (and which corresponds to some of the stars shown in the bottom left panel of Fig. 6).

\subsection{Ratio of the number of stars in the two $V_{Z}$ groups}

As mentioned in the introduction, the ratio of the number of stars in the two $V_{Z}$ groups was used in Kepley et al. (2007) to estimate the time of accretion of the object. The ratio these authors used
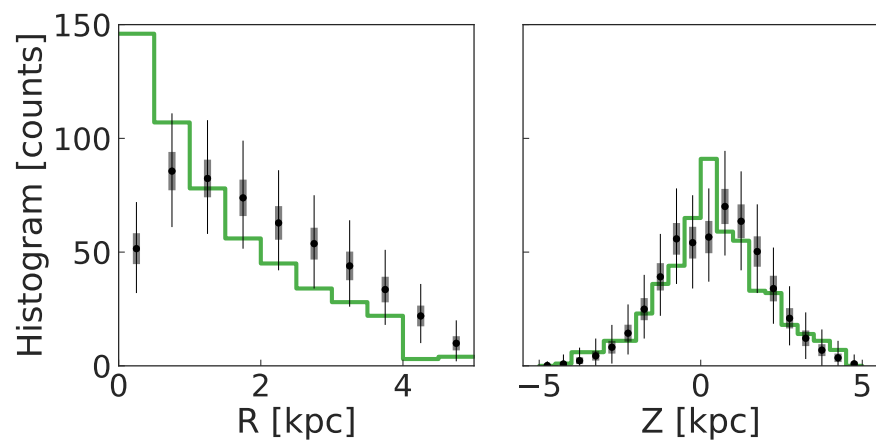

Fig. 5. Distribution of heliocentric cylindrical $R($ left $)$ and $Z(r i g h t)$ for stars in our $6 \mathrm{D}$ sample. The green histograms are for members of the Helmi streams. With black symbols, we show the mean counts obtained using $10^{4}$ random sets extracted from our (background) halo sample, with the grey and black error bars indicating the $1 \sigma$ and $3 \sigma$ uncertainties for each $R / Z$-bin. The Helmi streams are clearly more confined in the $R$-direction.

was 1:2, in good agreement with the ratio found here in Sect. 3.1 for the core members. Using numerical simulations, this implied an accretion time of 6-9 Gyr for an object of total dynamical mass of $\sim 4 \times 10^{8} M_{\odot}$. Now with a sample of up to 523 members, we will analyse how this ratio varies when exploring beyond the immediate solar vicinity.

Figure 8 shows the ratio of the number of stars in the two streams in $V_{Z}$ for selection $\mathrm{B}$, as a function of the extent of the volume considered. Blue indicates the ratio of all the stars and green is for the 6D Gaia-only sample. The central lines plot the measured ratio and the shaded areas correspond to the $1 \sigma$ Poissonian error, showing that there is good agreement between the samples. The dashed lines at 1:2, 3:5 and 2:5 encompass roughly the mean and the scatter in the ratio. Evidently, the ratio drops slightly beyond the $1 \mathrm{kpc}$ volume around the Sun, however, overall it stays rather constant and takes a value of $\approx 1: 2$ for stars with $V_{Z}>0$ relative to those with $V_{Z}<0$.

\subsection{HR diagram and metallicity information}

Photometry from Gaia combined with auxiliary metallicity information from the APOGEE/RAVE/LAMOST surveys can give us insights into the stellar populations of the Helmi streams. By using the Gaia parallaxes we construct the HertzsprungRussell (HR) diagram shown in Fig. 9. We have used here a sample of high photometric quality by applying the selection criteria from Arenou et al. (2018) and described in Sect. 3.3. Because the photometry in the BP passband is subject to some systematic effects especially for stars in crowded regions (see Gaia Collaboration 2018b), we use $\left(G-G_{\mathrm{RP}}\right)$ colour.

In Fig. 9 the streams' members from the 6D sample are plotted with green symbols, and in blue if they are from the 5D dataset. On the basis of a colour-colour diagram we have identified stars that are likely reddened by extinction, see Appendix A, and these are indicated with black dots. Since most stars follow a well-defined sequence in the $\left[\left(G-G_{\mathrm{RP}}\right),\left(G-G_{\mathrm{BP}}\right)\right]$ space, outliers can be picked out easily. We consider as outliers those stars with a $\left(G-G_{\mathrm{BP}}\right)$ offset greater than 0.017 from the sequence (i.e. $5 \times$ the mean error in the colours used). We find that especially the members found in the 5D dataset appear to be reddened. This is expected as all of these stars are located at low Galactic latitude (within $15^{\circ}$ from the galactic centre or anti-centre). Figure 9 shows also that we are biased towards finding relatively 
H. H. Koppelman et al.: Characterization and history of the Helmi streams with Gaia DR2
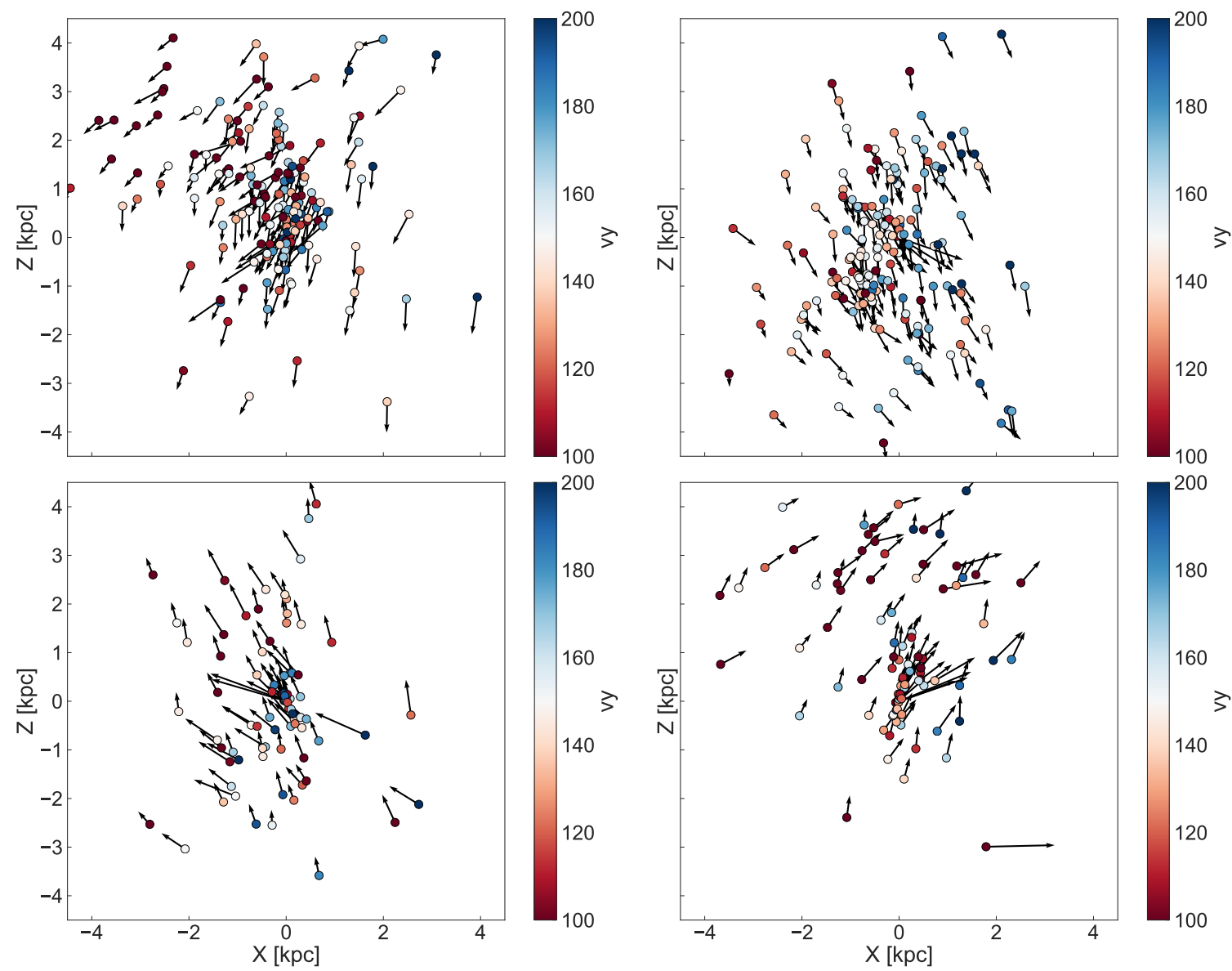

Fig. 6. Distribution of the stars in the Helmi streams in the $X Z$ plane (i.e. perpendicular to the disc of the Milky Way), with the arrows illustrating their motions (amplitude and direction) in this plane. The symbols are colour-coded according to the amplitude of their $V_{Y}$ velocity (i.e. perpendicular to the projected plane). Top and bottom rows: stream members with $V_{Z}<0$ and $V_{Z}>0$, respectively. Left and right columns: stars with $V_{X}<0$ and $V_{X}>0$, respectively. In all the four panels streaming motions and substructures are clearly apparent.

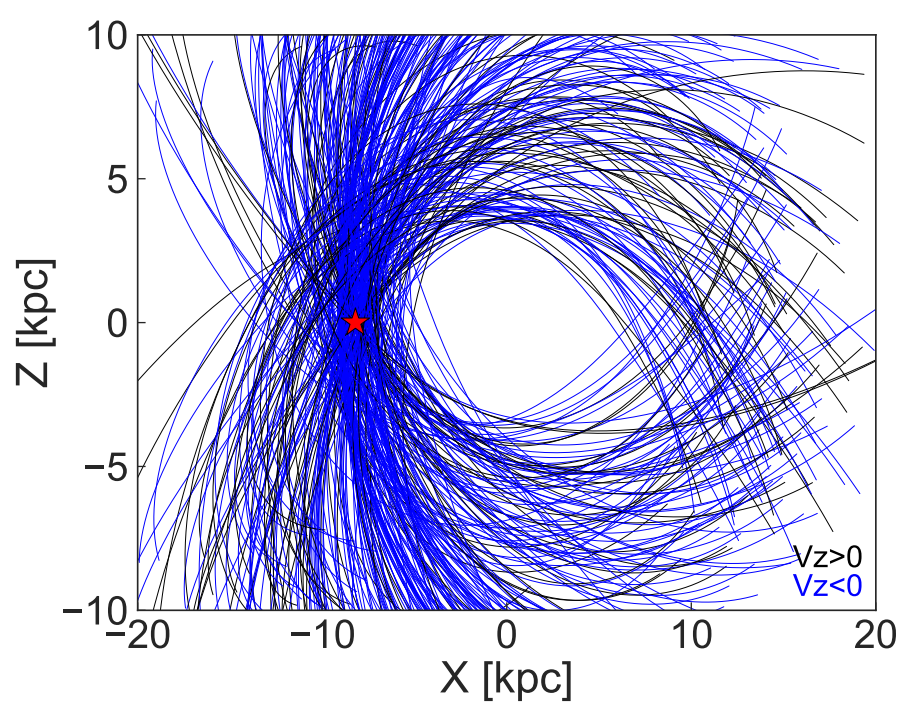

Fig. 7. Compilation of orbits based on the $6 \mathrm{D}$ positions of the members of the Helmi streams. These orbits have been integrated for $100 \mathrm{Myr}$ backwards and forward in time. The position of the Sun is illustrated with a red star, the Galactic centre is at $(X, Z)=(0,0)$ in this frame. The trajectories of stars that currently have $V_{Z}>0$ are coloured black, while those with $V_{Z}<0$ are shown in blue. Close to the solar position, the majority of the Helmi streams' members move perpendicular to the plane of the disc, and are close to pericentre.

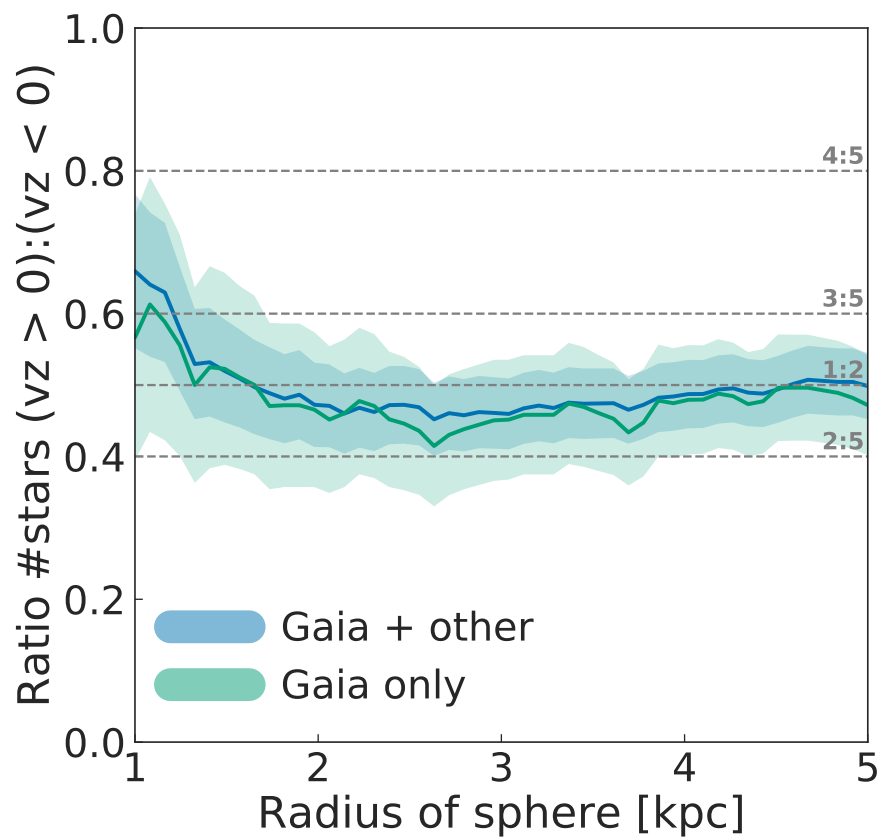

Fig. 8. Ratio of the number of stars in the two clumps in $V_{Z}$ using selection box $\mathrm{B}$, for different volumes and for the two samples of stars as indicated. The shaded area corresponds to the Poisson error on the measured ratio. 


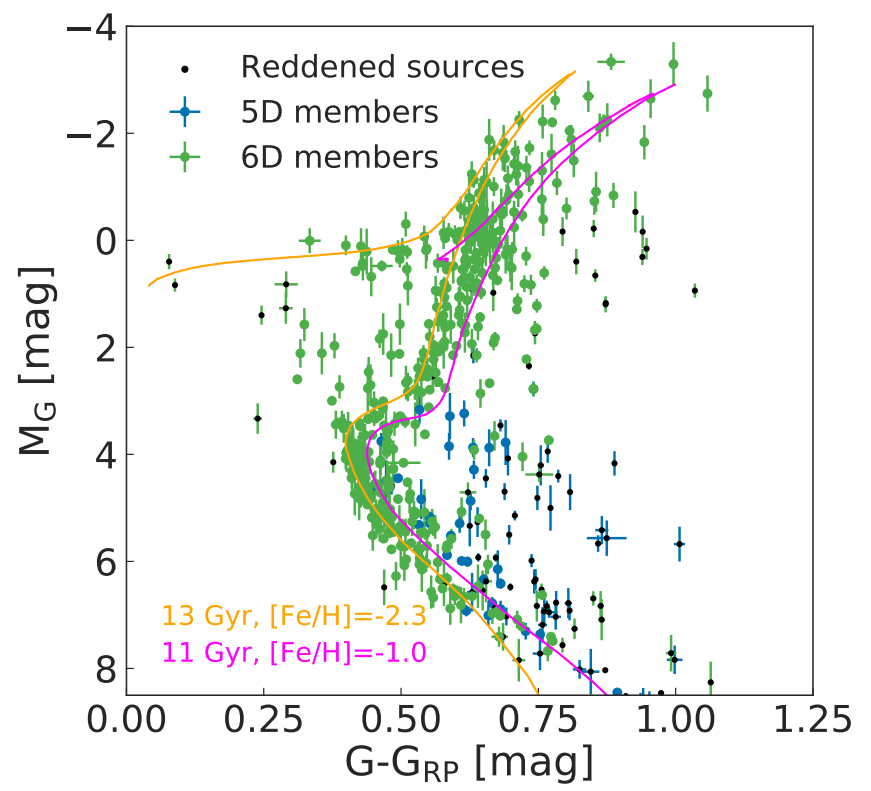

Fig. 9. Hertzsprung-Russel diagram of members of the Helmi streams. Those identified in the 6D sample are shown in green, while those without radial velocities are indicated with blue symbols. Members that are likely highly reddened are indicated with black dots, see Appendix A Superimposed are single population isochrones taken from Marigo et al. (2017). They serve to illustrate that the Helmi streams include a range of old, metal-poor stellar populations which did not form in a single event.

more intrinsically bright than fainter stars, and this is due to the quality cuts applied and to the magnitude limits of the samples used. We expect however that there should be many more fainter, lower main sequence stars that also belong to the Helmi streams, hidden in the local stellar halo.

The HR diagram that is shown in Fig. 9 does not resemble that of a single stellar population, but rather favours a wide stellar age distribution of $\sim 2$ Gyr spread, based on the width of the main sequence turn-off. To illustrate this we have overlayed two isochrones from Marigo et al. (2017) for single stellar populations of 11 and $13 \mathrm{Gyr}$ old age and with metallicities $[\mathrm{Fe} / \mathrm{H}]=-1.0$ and -2.3 respectively. To take into account the difference between the theoretical and actual Gaia passbands (Weiler 2018), we have recalibrated the isochrones on globular clusters with similar age and metallicity (NGC 104, NGC 6121, NGC 7099, see Harris 1996), which led to a shift in $\left(G-G_{\mathrm{RP}}\right)$ colour of $0.04 \mathrm{mag}$.

The spread in metallicities used for the isochrones is motivated by the metallicity distribution shown in Fig. 10, and derived using the stream members found in the APOGEE/ RAVE/LAMOST datasets. We have used the following metallicity estimates for the different surveys: Met_K for RAVE, FE_H for APOGEE and feh for LAMOST. The median errors for these quantities are: 0.17 dex for RAVE, 0.12 dex for LAMOST, and 0.02 for APOGEE. The distribution plotted in Fig. 10 shows a range of metallicities $[\mathrm{Fe} / \mathrm{H}]=[-2.3,-1.0]$, with a peak at $[\mathrm{Fe} / \mathrm{H}]=-1.5$. The small tail seen towards the metal-rich end, that is at $[\mathrm{Fe} / \mathrm{H}] \sim-0.5$ is likely caused by contamination from the thick disc. The shape of the distribution shown in Fig. 10 is reminiscent of that reported by Klement et al. (2009) and by Smith et al. (2009) for much smaller samples of members of the Helmi streams. Roederer et al. (2010) have carried out detailed abundance analysis of the original streams' members which confirm the range of $\sim 1 \mathrm{dex}$ in $[\mathrm{Fe} / \mathrm{H}]$ found here. All this evi-

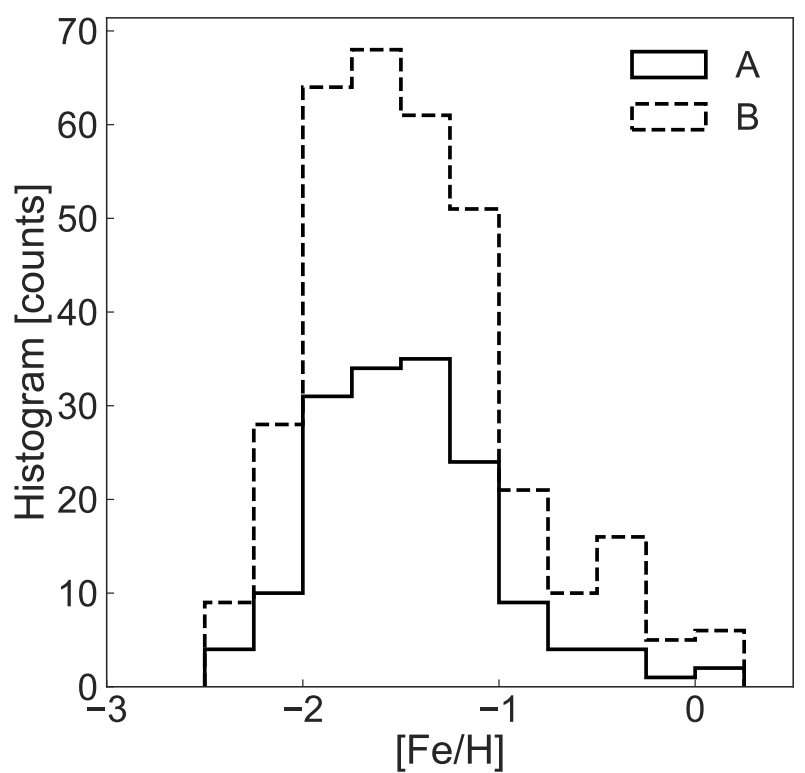

Fig. 10. Histogram of the metallicities of the Helmi streams stars that are in the APOGEE/RAVE/LAMOST datasets. The distributions are very similar for selections $\mathrm{A} \& \mathrm{~B}$, peaking at $[\mathrm{Fe} / \mathrm{H}] \sim-1.5$ and revealing a broad range of metallicities for the Helmi streams stars. The metalrich tail $([\mathrm{Fe} / \mathrm{H}] \sim-0.5)$ is likely due to contamination from the thick disc but it is minimal for both selection boxes.

Table 2. Structural parameters of the simulated dwarf galaxies.

\begin{tabular}{ccccc}
\hline & prog. 1 & prog. 2 & prog. 3 & prog. 4 \\
\hline$M_{*}\left(M_{\odot}\right)$ & $5 \times 10^{6}$ & $10^{7}$ & $5 \times 10^{7}$ & $10^{8}$ \\
$r_{\mathrm{s}, *}(\mathrm{kpc})$ & 0.164 & 0.207 & 0.414 & 0.585 \\
$r_{\mathrm{s}, \mathrm{NFW}}(\mathrm{kpc})$ & 1.32 & 1.72 & 3.42 & 4.26 \\
$M_{\mathrm{dm}}\left(M_{\odot}\right)$ & $5 \times 10^{8}$ & $10^{9}$ & $5 \times 10^{9}$ & $10^{10}$ \\
$M_{\mathrm{dm}, \text { trunc }}\left(M_{\odot}\right)$ & $1.9 \times 10^{8}$ & $3.8 \times 10^{8}$ & $1.84 \times 10^{9}$ & $3.62 \times 10^{9}$ \\
$r_{\mathrm{c}, \text { trunc }}(\mathrm{kpc})$ & 2.0 & 2.5 & 4.1 & 5 \\
$r_{\mathrm{d}, \text { trunc }}(\mathrm{kpc})$ & 0.65 & 0.86 & 1.62 & 2.13 \\
\hline
\end{tabular}

Notes. We quote both the dark halo's original and truncated mass. This truncation depends on two parameters: $r_{\mathrm{c}, \text { trunc }}$ and $r_{\mathrm{d} \text {,trunc }}$, the cut-off and the decay radii, respectively.

dence bolsters our claim that the streams originate in an object that had an extended star formation history.

\section{Simulating the streams}

We focus here on N-body experiments which we carried out to reproduce some of the properties of the Helmi streams. To this end, we used a modified version of GADGET 2 (Springel et al. 2005) that includes the rigid, static host potential described in Sect. 3.2 to model the Milky Way.

The analysis presented in previous sections, and in particular the HR diagram and metallicity distribution of member stars, supports the hypothesis that the Helmi streams stem from a disrupted (dwarf) galaxy. We therefore model the progenitor of the streams as a dwarf galaxy with a stellar and a dark matter component. We consider four possible progenitors whose characteristics are listed in Table 2.

For the stellar component, we use $10^{5}$ particles distributed following a Hernquist profile, whose structural properties are motivated by the scaling relations observed for dwarf spheroidal galaxies (Tolstoy et al. 2009). For the dark matter halo we use 

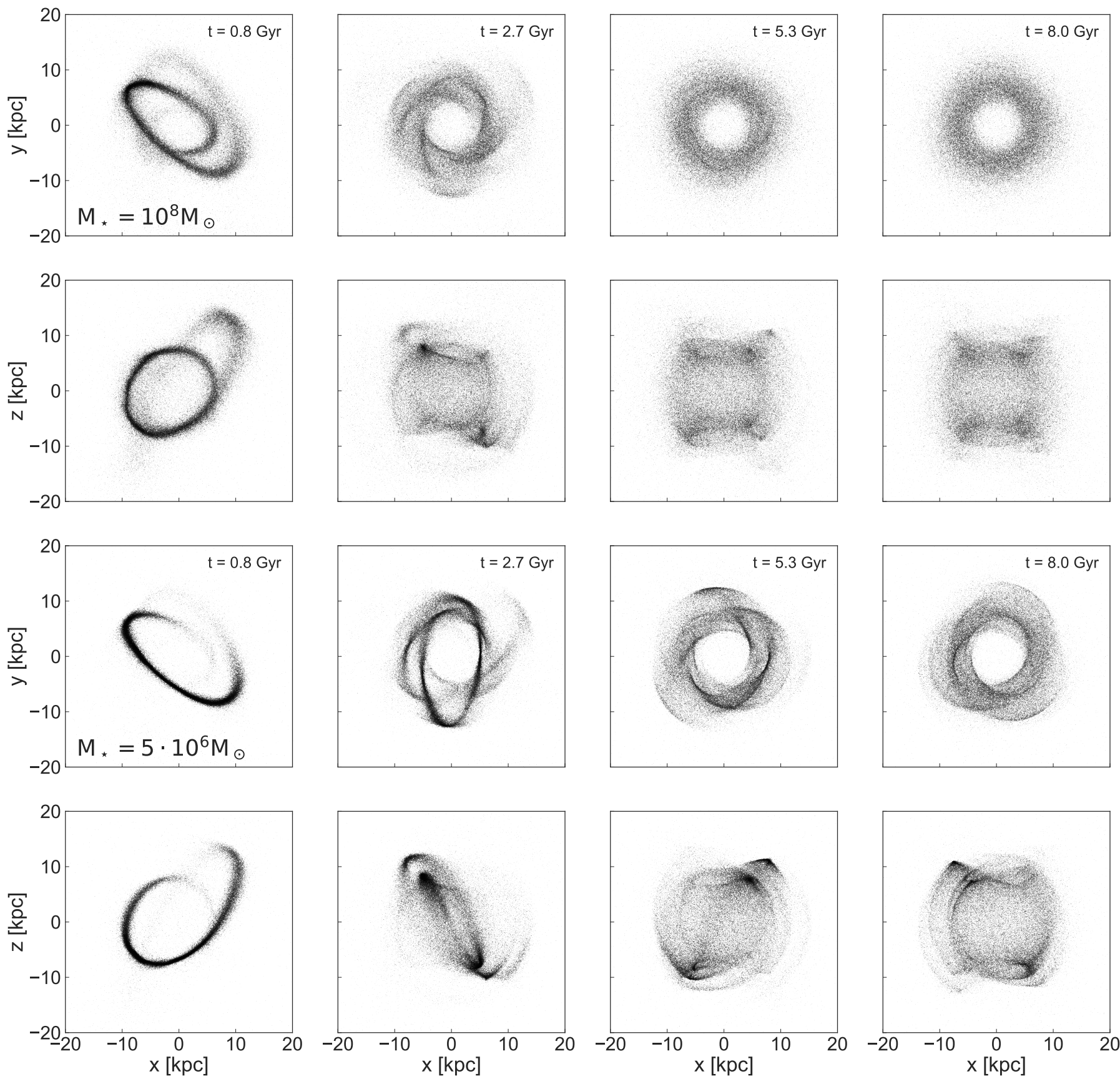

Fig. 11. Spatial evolution of the Helmi streams at four different snapshots in our simulations. The top two rows show the evolution of a progenitor with a stellar mass of $10^{8} M_{\odot}$, while the bottom two rows correspond to a system with a stellar mass of $5 \times 10^{6} M_{\odot}$. In both cases, the orbit of the progenitor is the same. The appearance of the debris is seen to depend on the time since accretion as well as on the mass of the progenitor.

$6 \times 10^{5}$ particles following a truncated NFW profile (similar to the model introduced in Springel \& White 1999, but where the truncation radius $r_{\mathrm{c} \text {,trunc }}$ and the decay radius $r_{\mathrm{d} \text {,trunc }}$ are specified independently), with characteristic parameters taken from Correa et al. (2015). We truncate the NFW halo at a radius where its average density is three times that of the host (at the orbital pericentre). After setting the system up using the methods described in Hernquist (1993), we let it relax for 5 Gyr in isolation. We then place it on an orbit around the Milky Way. This orbit is defined by the mean position and velocity of the stars that were identified as core members of the stream with $V_{Z}<0^{3}$.

\footnotetext{
3 We take the $V_{Z}<0$ clump as this has the largest number of members.
}

Figure 11 illustrates the evolution of a high-mass dwarf galaxy in the top two rows, and a low-mass dwarf galaxy in the bottom two rows. In each panel, we show the star particles in galactocentric Cartesian coordinates for different times up to $8 \mathrm{Gyr}$ after infall. This comparison shows that increasing the mass results in more diffuse debris. On the other hand, the time since accretion has an impact on the length of the streams and on how many times the debris wraps around the Milky Way.

\subsection{Estimation of the mass and time of accretion}

To constrain the history of the progenitor of the Helmi streams we use the ratio of the number of stars in the two clumps in 


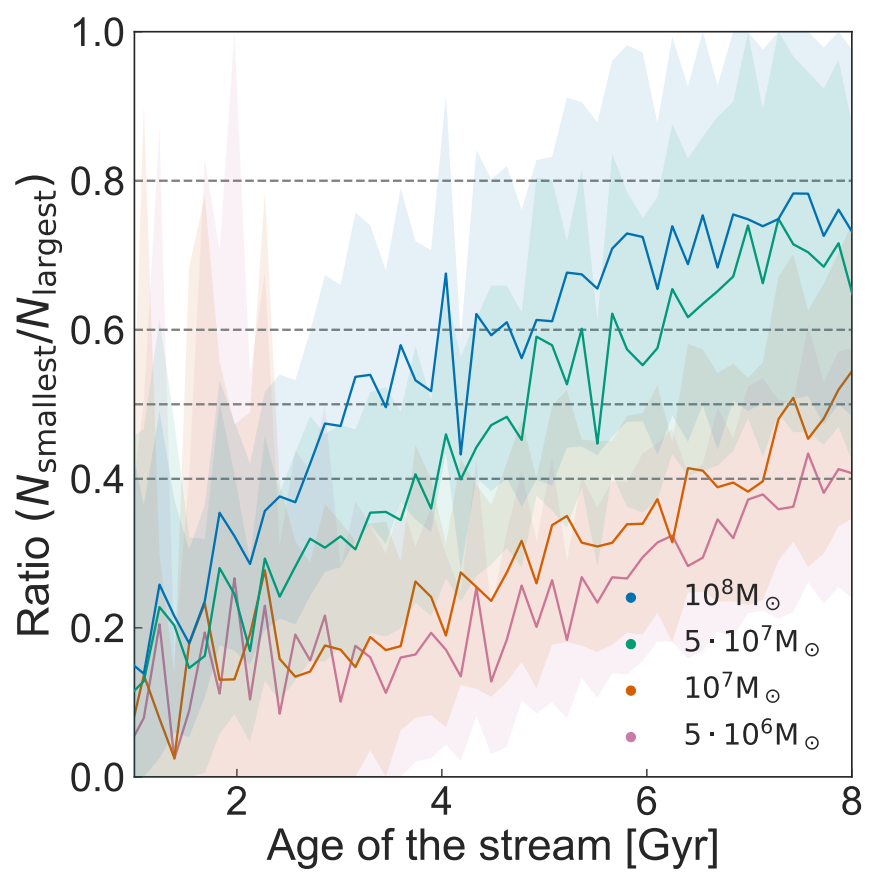

Fig. 12. Ratio of the numbers of stars in the less populated $V_{Z}$ clump compared to that of the more populated clump, as a function of time for our N-body simulations. The solid lines show the mean values, averaged over 25 different volumes spread uniformly along a circle of $1 \mathrm{kpc}$ at the solar radius.

$V_{Z}$ as well as their velocity dispersion. Typically, the simulated streams do not have a uniform spatial distribution and they show variations on small scales. Furthermore, the azimuthal location of the Sun in the simulations is arbitrary. Therefore, and also to even out some of the small-scale variations, we measure the ratio of the number of stars in the streams and their velocity dispersion in 25 volumes of $1 \mathrm{kpc}$ radius located at $8.2 \mathrm{kpc}$ distance from the centre, and distributed uniformly in the azimuthal angle $\phi$.

Figure 12 shows the mean of the ratio for the different volumes as a function of time with solid curves, with the colours marking the different progenitors listed in Table 2. The shaded areas correspond to the mean Poissonian error in the measured ratio for the different volumes. The horizontal dashed lines are included for guidance and correspond to the lines shown in Fig. 8 for the actual data.

The mean ratio is clearly correlated with the properties of the progenitor, the most massive one being first to produce multiple streams in a given volume. Massive satellites have a larger size and velocity dispersion, which causes them to phase mix more quickly because of the large range of orbital properties (energies, frequencies). Based solely on Fig. 12, and taking a ratio between 0.55 and 0.7 as found using the Gaia-only sample in a $1 \mathrm{kpc}$ sphere, we would claim that there is a range of possible ages of the stream, with the youngest being $\sim 4.5 \mathrm{Gyr}$ for the most massive progenitor, while for the lowest mass object the age would have to be at least $8 \mathrm{Gyr}$.

A different way of probing the properties of the progenitor is to use the velocity dispersion of the streams. In Fig. 13 we show the dispersion along two principal axes of the velocity ellipsoid for the $1 \mathrm{kpc}$ volumes that satisfy the ratio-constraint on the number of stars in the two $V_{Z}$ clumps, with open/closed markers used for the least/most populated clump. Green markers indicate the measured velocity dispersions. These dispersions are deconvolved of their errors, and calculated after clipping $1 \sigma$ outliers in $V_{\mathrm{R}}$ (see next paragraph). Typically the simulations
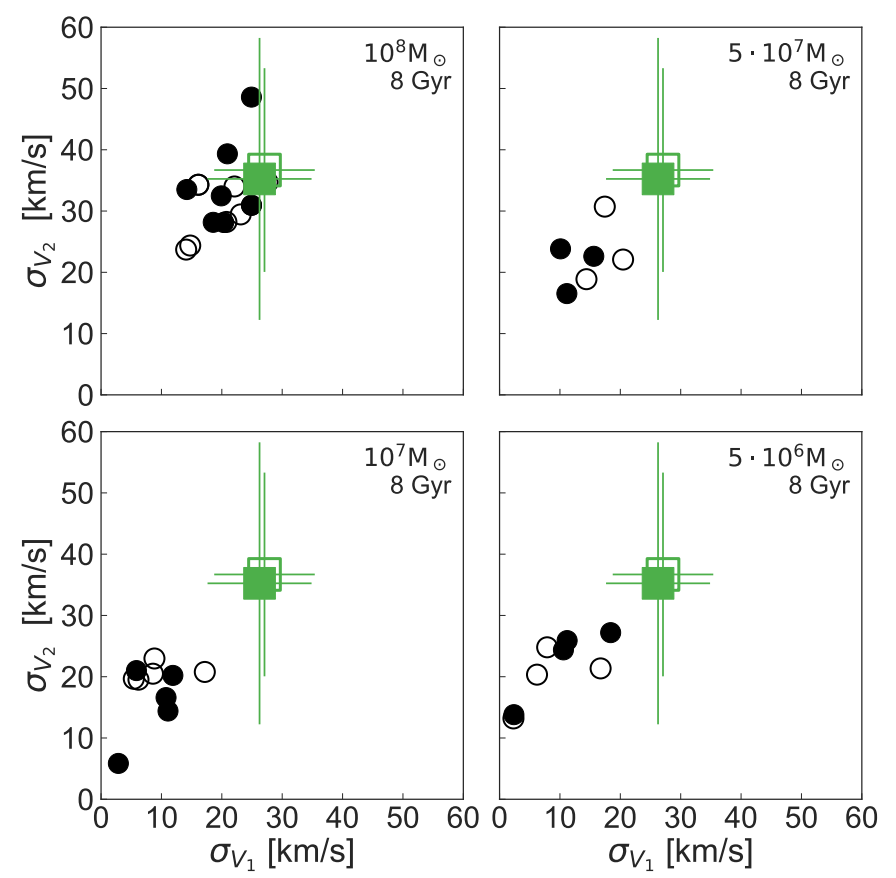

Fig. 13. Velocity dispersions of the simulated streams 8 Gyr after accretion. These have been computed using the principal axis of the velocity ellipsoid of stars in a given $V_{Z}$ clump, open markers are used for the smallest clump and closed markers for the largest. We show the results for volumes satisfying the ratio in the number of stars in the clumps as observed for the data. The green markers indicate the measured velocity dispersion from the data, the error bars illustrate the scatter in 1000 randomly down sampled sets of the streams members. These measured dispersions are deconvolved of their errors, and a $1 \sigma$ clip in $V_{\mathrm{R}}$ is applied.

have fewer particles in such $1 \mathrm{kpc}$ volumes than observed in the debris. Therefore, we down-sample the data to only 15 stars per stream, which corresponds to the average number of particles in the simulations. The error bars indicate the maximum scatter in the velocity dispersion for 1000 such random down sampled sets.

The velocity ellipsoid of the streams' members is roughly aligned in cylindrical coordinates with $V_{1}, V_{2}$, and $V_{3}$ corresponding respectively to $V_{\phi}, V_{Z}, V_{\mathrm{R}}$. We note that only for the most massive progenitor (and for times $>5 \mathrm{Gyr}$ ) the velocity dispersions are in good agreement with the observed values for $\sigma_{V 1}$ and $\sigma_{V 2}$, and that in all the remaining simulations, the dispersions are too small compared to the data. We should point out however, that the largest velocity dispersion (i.e. that along $V_{R}$ ) is less well reproduced in our simulations, possibly indicating that the range of energies of the debris is larger. However, if we clip $1 \sigma$ outliers in $V_{\mathrm{R}}$ for the data, and recompute $\sigma_{V 3}$, we find much better agreement with the simulations (while the values of $\sigma_{V 1}$ and $\sigma_{V 2}$ remain largely the same). The clipped members of the Helmi streams are all in the high energy, $E$, tail (see Fig. 2), suggesting perhaps that the object has suffered some amount of dynamical friction in its evolution.

Combining the information of the ratio (Fig. 12) and the corresponding velocity dispersion measurements (Fig. 13) we therefore conclude that the most likely progenitor of the Helmi streams was a massive dwarf galaxy with a stellar mass of $\sim 10^{8} M_{\odot}$. In general, the simulations suggest a range of plausible accretion times from 5-8 Gyr. Although these estimates of the time of accretion are different than those obtained by Kepley et al. (2007), our results are consistent when a similar 

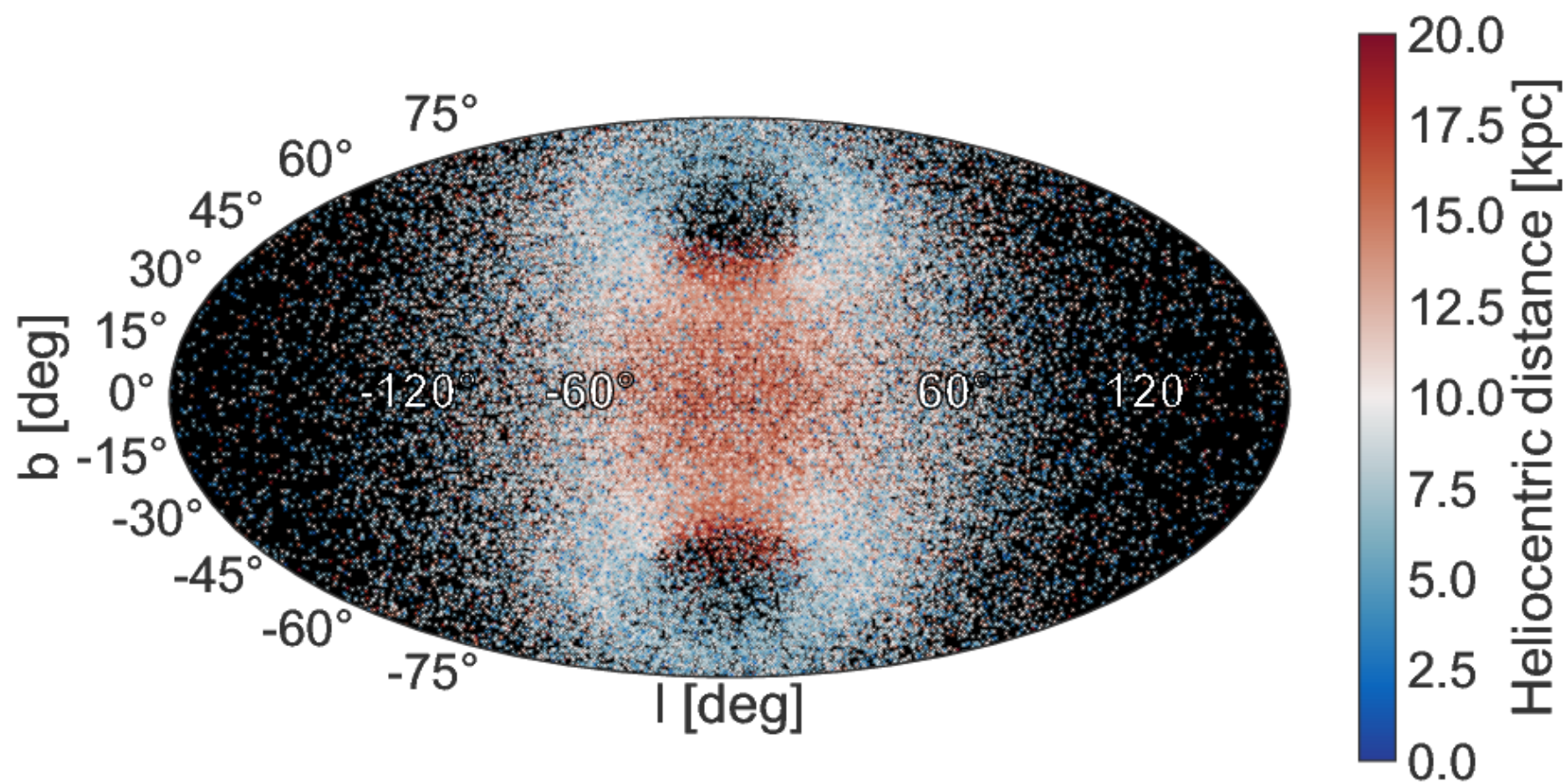

Fig. 14. Mollweide projection in Galactic sky coordinates $l$ and $b$ of the distribution of star particles for the simulation of a progenitor dwarf galaxy with a stellar mass of $10^{8} M_{\odot}$. The colour-coding gives the heliocentric distance of the particles.

progenitor mass is used (i.e. of models 1 and 2), which however, does not reproduce well the observed kinematical properties of the streams.

\subsection{Where to find new members across the Milky Way}

To investigate where to find new members of the Helmi streams beyond the solar neighbourhood we turn to the simulations.

In Fig. 14 we show a sky map of stars from the N-body simulation corresponding to the dwarf galaxy with $M_{*}=10^{8} M_{\odot}$, 8.0 Gyr after accretion (same as shown in the top right panel of Fig. 11). The coordinates shown here are galactic $l$ and $b$ plotted in a Mollweide projection with the Galactic centre located in the middle (at $l=0$ ). Nearby stars (in blue) are mainly distributed along a "polar ring-like" structure between longitudes $\pm 60^{\circ}$ (see for comparison Fig. 6). The most distant members are found behind the bulge (in red), but because of their location they may be difficult to observe.

\section{Association with globular clusters}

If the progenitor of the Helmi streams was truly a large dwarf galaxy, it likely had its own population of globular clusters (see Leaman et al. 2013; Kruijssen et al. 2018). To this end we look at the distribution of the debris in IOM-space for the simulation of the progenitor with $M_{*}=10^{8} M_{\odot}$ and overlay the data for the globular clusters from Gaia Collaboration (2018c).

Figure 15 shows the energy $E$ vs $L_{z}$ (top) and the $L_{\perp}$ vs $L_{z}$ (bottom) of star particles in the simulation (black) and the stream members (green) together with the globular clusters (white open circles). For easy comparison we have overplotted the same red selection boxes as those in Fig. 2. We have labelled the globular clusters that show overlap with the streams members in this space. Those that could tentatively be associated on the basis of their orbital properties are: NGC 4590 ,
NGC 5024, NGC 5053, NGC 5272, NGC 5634, NGC 5904, and NGC 6981.

This set of globular clusters shows a moderate range in age and metallicity: they are all old with ages $\sim 11-12 \mathrm{Gyr}$ and metal-poor with metallicities $[\mathrm{Fe} / \mathrm{H}]=[-2.3,-1.5]$. These age estimates are from Vandenberg et al. (2013), while for NGC 5634 we set it to 12 Gyr from comparison to NGC 4590 based on the zero-age HB magnitude (Bellazzini et al. 2002), and assume an uncertainty of 0.5 Gyr. Figure 16 shows the agemetallicity relation of all globular clusters that have reliable ages with those that we have associated with the Helmi streams coloured green. Interestingly the Helmi streams' clusters follow a relatively tight age-metallicity relation, and which is similar to that expected if they originate in a progenitor galaxy of $M_{*} \sim 10^{7}-10^{8} M_{\odot}$ (see Leaman et al. 2013 Fig. 4).

Figure 17 shows the Gaia colour-magnitude diagrams (CMD) of the globular clusters tentatively associated with the Helmi streams. Although not all CMDs are well-populated because of limitations of the Gaia DR2 data, their properties do seem to be quite similar, increasing even further the likelihood of their association to the progenitor of the Helmi streams.

Some of the associated globular clusters, namely NGC 5272, NGC 5904, and NGC 6981 have in fact, been suggested to have an accretion origin (of a yet unknown progenitor, see Kruijssen et al. 2018, and references therein). Two other clusters, NGC 5024 and NGC 5053, have at some point been linked to Sagittarius, although recent proper motion measurements have demonstrated this association is unlikely (see Law \& Majewski 2010; Sohn et al. 2018; Gaia Collaboration 2018c). Also NGC 5634 has been related to Sagittarius (Law \& Majewski 2010; Carretta et al. 2017) based on its position and radial velocity. However, the proper motion of the system measured by Gaia Collaboration (2018c) is very different from the prediction by for example, the Law \& Majewski (2010) model of the Sagittarius streams. 

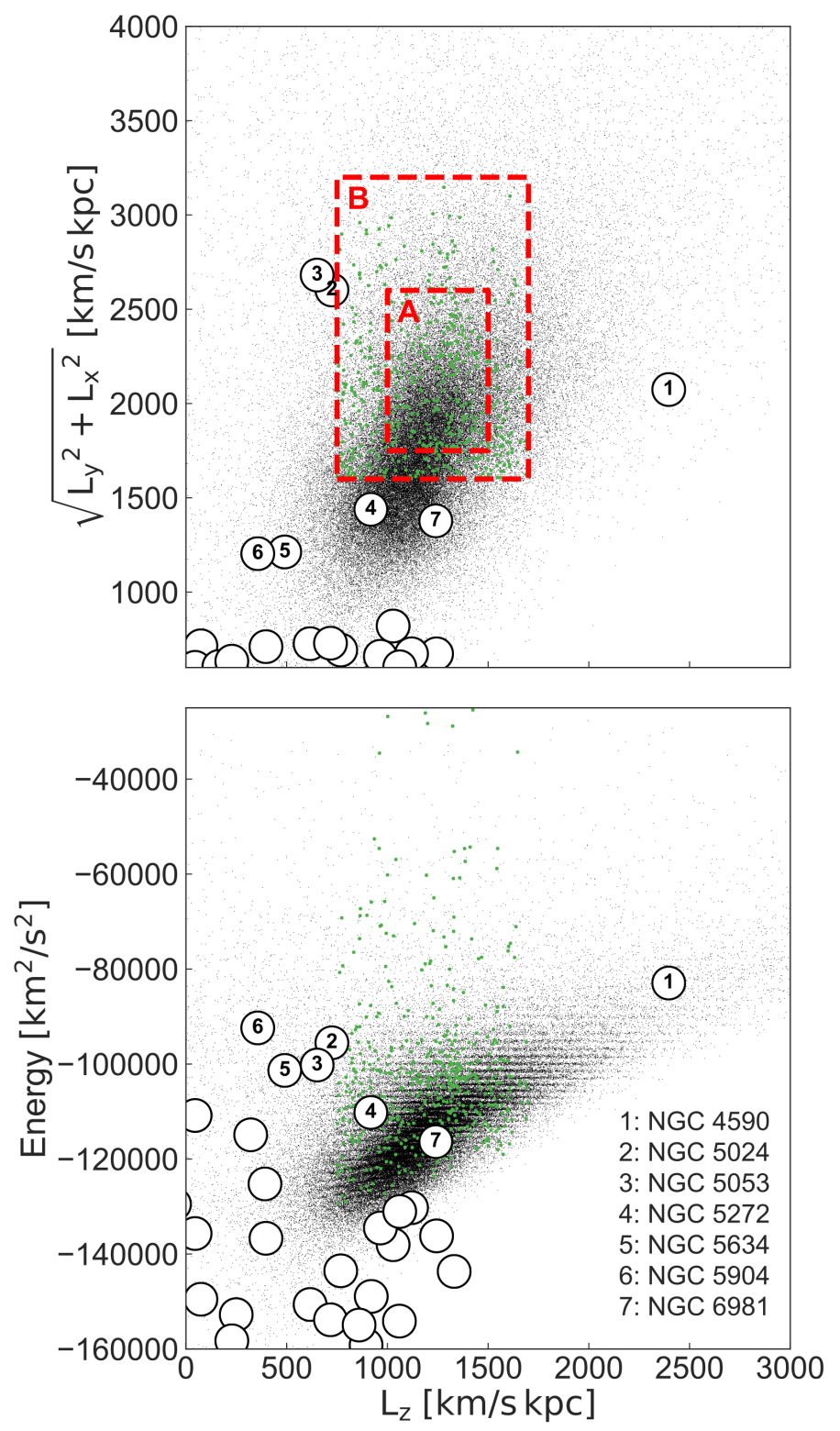

Fig. 15. $L_{\perp}-L_{z}$ (bottom) and the $E-L_{z}$ (top) distributions of the simulated stars (in black), together with the selected $6 \mathrm{D}$ stream members (in green). Plotted here are the star particles from the simulation of the most massive dwarf, with a stellar mass of $10^{8} M_{\odot}$ and accretion time of $8.0 \mathrm{Gyr}$. Large symbols indicate the location of Milky Way globular clusters. Those possibly associated with the Helmi streams based on their location in these panels are numbered.

\section{Conclusions}

Using the latest data from Gaia DR2 combined with the APOGEE/RAVE/LAMOST surveys we find hundreds of new tentative members of the Helmi streams. In the 6D sample that we built, we identified 523 members on the basis of their orbital properties, in particular their energy and angular momenta. On the other hand, we found 105 stars in the full Gaia 5D dataset in two $15^{\circ}$-radius fields around the galactic centre and anticentre, using only the tangential velocities of the stars (which translate directly into two components of their angular momenta). Despite the large number of newly identified members we expect that many, especially faint stars are still hiding, even within a volume of $1 \mathrm{kpc}$ around the Sun.

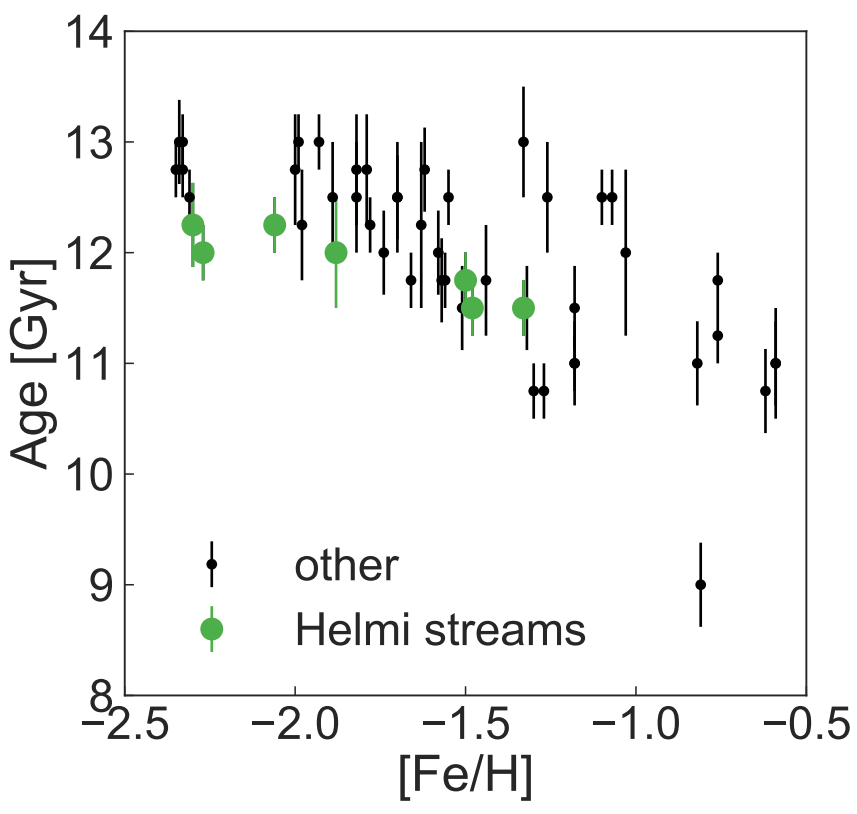

Fig. 16. Age-metallicity distribution of Milky Way globular clusters based on Vandenberg et al. (2013). The green symbols mark the clusters associated with the Helmi streams on the basis of their orbital properties. They follow a well-defined age-metallicity relation.

Having such an unprecedented sample of members of the streams allows us to characterize the streams and the nature of their progenitor. The HR diagram of the members suggests an age range of $\sim 11-13 \mathrm{Gyr}$, while their metallicity distribution goes from $[\mathrm{Fe} / \mathrm{H}] \sim-2.3$ to -1.0 , with a peak at $[\mathrm{Fe} / \mathrm{H}] \sim-1.5$. We are also able to associate to the streams seven globular clusters on the basis of their dynamical properties. These clusters have similar ages and metallicities as the stars in the streams. Remarkably they follow a well-defined age-metallicity relation, and similar to that expected for clusters originating in a progenitor galaxy of $M_{*} \sim 10^{7}-10^{8} M_{\odot}$ (Leaman et al. 2013).

This relatively high value of the stellar mass is also what results from $\mathrm{N}$-body simulations that aim to recreate the observed dynamical properties of the streams. From the ratio of the number of stars in the two clumps in $V_{Z}$ and their velocity dispersion we estimate the time of accretion to be in the range 5-8 Gyr and a stellar mass for the dwarf galaxy of $\sim 10^{8} \mathrm{M}_{\odot}$.

Although 5 Gyr ago would imply a relatively recent accretion event, one might argue that the object was probably on a less bound orbit and sunk in via dynamical friction (thanks to its large mass) and started to get disrupted then. This could explain the mismatch between the age of the youngest stars in the streams $(\approx 11 \mathrm{Gyr}$ old $)$, and the time derived dynamically.

Despite the fact that the simulations are able to recreate the observations reasonably well, they fail to reproduce fully the observed velocity distribution in particular in the radial direction. This could be due to the lack of dynamical friction, but also by the limited exploration of models for the potential of the Milky Way. Other important improvements will be to consider the inclusion of gas particles and star formation in the simulations, as well as different initial morphologies for the progenitor systems (not only spherical, but also disc-like).

Originally, $\mathrm{H} 99$ determined that $10 \%$ of the stellar halo mass beyond the solar radius could belong to the progenitor of the Helmi streams. The lack of a significant increase in the number of members subsequently discovered by other groups (Chiba $\&$ Beers 2000), led to the suggestion that the fraction may be 

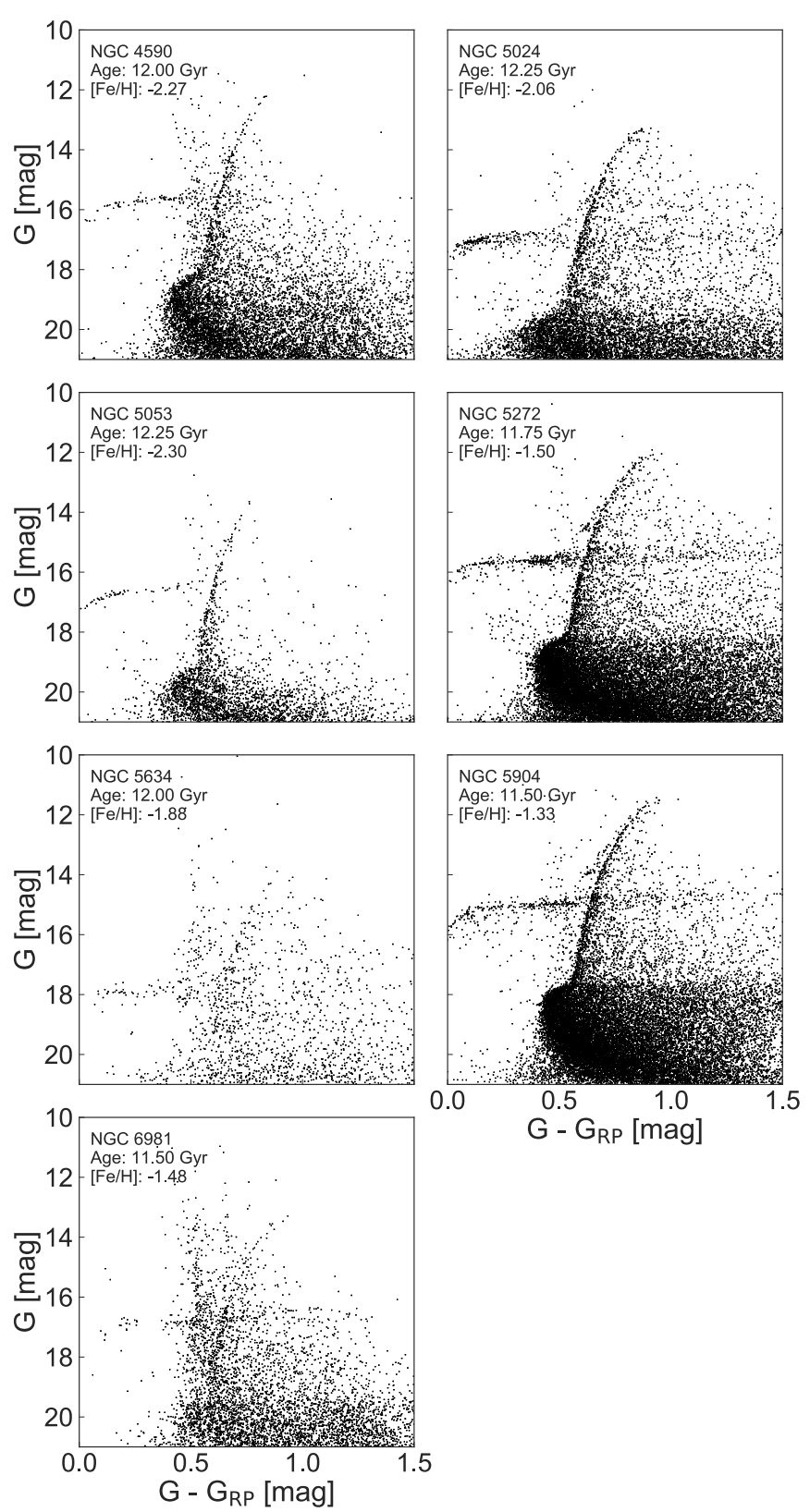

$\mathrm{G}-\mathrm{G}_{\mathrm{RP}}[\mathrm{mag}]$

Fig. 17. Gaia colour-magnitude diagrams for the globular clusters that are likely associated with the Helmi streams on the basis of their orbital properties. All the CMDs correspond to stellar populations that are old and metal-poor. The ages and metallicities shown are taken from Vandenberg et al. (2013), except for the age of NGC 4590, which is based on the zero-age HB magnitude (Bellazzini et al. 2002).

lower. Our best estimate of the stellar mass of the progenitor of the Helmi streams is $\sim 10^{8} M_{\odot}$, implying that it does significantly contribute to the stellar halo. For example Bell et al. (2008) estimate a stellar mass for the halo of $(3.7 \pm 1.2) \times 10^{8} M_{\odot}$ between galactocentric radii of 1 to $40 \mathrm{kpc}$, and hence being a lower limit to the total stellar halo mass. Other estimates, based on the local density of halo stars give $7-10 \times 10^{8} M_{\odot}$ (see Morrison 1993; Bland-Hawthorn \& Gerhard 2016). This implies that the Helmi streams may have contributed $\sim 10-14 \%$ of the stars in the Galactic halo.

Acknowledgements. We gratefully acknowledge financial support from a VICI grant from the Netherlands Organisation for Scientific Research (NWO) and from NOVA. This work has made use of data from the European Space Agency (ESA) mission Gaia (http: //www . cosmos. esa.int/gaia), processed by the Gaia Data Processing and Analysis Consortium (DPAC, http://www . cosmos. esa.int/web/gaia/dpac/consortium). Funding for the DPAC has been provided by national institutions, in particular the institutions participating in the Gaia Multilateral Agreement. We have also made use of data from: (1) the APOGEE survey, which is part of Sloan Digital Sky Survey IV. SDSS-IV is managed by the Astrophysical Research Consortium for the Participating Institutions of the SDSS Collaboration (http: //www . sdss . org); (2) the RAVE survey (http://www.rave-survey.org), whose funding has been provided by institutions of the RAVE participants and by their national funding agencies; (3) the LAMOST DR4 dataset, funded by the National Development and Reform Commission. LAMOST is operated and managed by the National Astronomical Observatories, Chinese Academy of Sciences. For the analysis, the following software packages have been used: vaex (Breddels \& Veljanoski 2018), numpy (Van Der Walt 2011), matplotlib (Hunter 2007), jupyter notebooks (Kluyver et al. 2016).

\section{References}

Abolfathi, B., Aguado, D. S., Aguilar, G., et al. 2018, ApJS, 235, 42 Anguiano, B., Majewski, S. R., Allende-Prieto, C., et al. 2018, A\&A, 620, 12 Arenou, F., Luri, X., Babusiaux, C., et al. 2018, A\&A, 616, 29 Beers, T. C., \& Sommer-Larsen, J. 1995, ApJS, 96, 175 Beers, T. C., Placco, V. M., Carollo, D., et al. 2017, ApJ, 835, 22 Bell, E. F., Zucker, D. B., Belokurov, V., et al. 2008, ApJ, 680, 295 Bellazzini, M., Ferraro, F. R., \& Ibata, R. 2002, ApJ, 124, 915

Belokurov, V., Zucker, D. B., Evans, N. W., et al. 2006, ApJ, 642, L137

Belokurov, V., Erkal, D., Evans, N. W., Koposov, S. E., \& Deason, A. J. 2018, MNRAS, 478, 611

Bernard, E. J., Ferguson, A. M. N., Schlafly, E. F., et al. 2016, MNRAS, 463, 1759

Bland-Hawthorn, J., \& Gerhard, O. 2016, ARA\&A, 54, 529

Bonaca, A., Conroy, C., Wetzel, A., Hopkins, P. F., \& Kereš, D. 2017, ApJ, 845, 101

Breddels, M. A., \& Veljanoski, J. 2018, A\&A, 618, 13

Bullock, J. S., \& Johnston, K. V. 2005, ApJ, 635, 931

Carretta, E., Bragaglia, A., Lucatello, S., et al. 2017, A\&A, 600, 118

Chiba, M., \& Beers, T. C. 2000, ApJ, 119, 2843

Chiba, M., \& Yoshii, Y. 1998, AJ, 115, 168

Cooper, A. P., Cole, S., Frenk, C. S., et al. 2010, MNRAS, 406, 744

Correa, C. A., Stuart, J., Wyithe, B., Schaye, J., \& Duffy, A. R. 2015, MNRAS, 452, 1217

Cui, X.-Q., Zhao, Y.-H., Chu, Y.-Q., et al. 2012, Res. Astron. Astrophys., 12, 1197

Gaia Collaboration (Babusiaux, C., et al.) 2018a, A\&A, 616, A10

Gaia Collaboration (Brown, A. G. A., et al.) 2018b, A\&A, 616, A1

Gaia Collaboration (Helmi, A., et al.) 2018c, A\&A, 616, A12

Gaia Collaboration (Katz, D., et al.) 2018d, A\&A, 616, A11

Grillmair, C. J. 2009, ApJ, 693, 1118

Grillmair, C. J., \& Dionatos, O. 2006, ApJ, 643, L17

Harris, W. E. 1996, AJ, 112, 1487

Haywood, M., Di Matteo, P., Lehnert, M. D., et al. 2018, ApJ, 863, 113

Helmi, A., \& de Zeeuw, P. T. 2000, MNRAS, 319, 657

Helmi, A., \& White, S. D. M. 1999, MNRAS, 307, 495

Helmi, A., White, S. D. M., De Zeeuw, P. T., \& Zhao, H. 1999, Nature, 402, 53

Helmi, A., White, S. D. M., \& Springel, V. 2003, MNRAS, 339, 834

Helmi, A., Navarro, J. F., Nordström, B., et al. 2006, MNRAS, 365, 1309

Helmi, A., Veljanoski, J., Breddels, M. A., Tian, H., \& Sales, L. V. 2017, A\&A, 598, A58

Helmi, A., Babusiaux, C., Koppelman, H. H., et al. 2018, Nature, 563, 85

Hernquist, L. 1993, ApJS, 86, 389

Hunter, J. D. 2007, Comput. Sci. Eng., 9, 90

Ibata, R. A., Gilmore, G., \& Irwin, M. J. 1994, Nature, 370, 194

Johnston, K. V., Hernquist, L., \& Bolte, M. 1996, ApJ, 465, 278

Kepley, A. A., Morrison, H. L., Helmi, A., et al. 2007, ApJ, 134, 1579

Klement, R., Fuchs, B., \& Rix, H.-W. 2008, ApJ, 685, 261

Klement, R., Rix, H.-W., Flynn, C., et al. 2009, ApJ, 698, 865

Kluyver, T., Ragan-Kelley, B., Pérez, F., et al. 2016, Jupyter Notebooks-a

Publishing Format for Reproducible Computational Workflows (IOS Press)

Koppelman, H., Helmi, A., \& Veljanoski, J. 2018, ApJ, 860, L11

Kruijssen, D. J. M., Pfeffer, J. L., Reina-Campos, M., Crain, R. A., \& Bastian, N. 2018, MNRAS, 1

Kunder, A., Kordopatis, G., Steinmetz, M., et al. 2017, ApJ, 153, 30

Law, D. R., \& Majewski, S. R. 2010, ApJ, 718, 1128

Leaman, R., VandenBerg, D. A., \& Mendel, J. T. 2013, MNRAS, 436, 122

Lindegren, L., Hernández, J., Bombrun, A., et al. 2018, A\&A, 616, A2 
Majewski, S. R., Nidever, D. L., Smith, V. V., et al. 2012, ApJ, 747, L37

Malhan, K., Ibata, R. A., \& Martin, N. F. 2017, MNRAS, 1

Marigo, P., Girardi, L., Bressan, A., et al. 2017, ApJ, 835, 19

Marrese, P. M., Marinoni, S., Fabrizio, M., \& Altavilla, G. 2018, A\&A, 621, A144

Massari, D., Posti, L., Helmi, A., Fiorentino, G., \& Tolstoy, E. 2017, A\&A, 598,

Mcmillan, P. J. 2017, MNRAS, 465, 76

Mcmillan, P. J., \& Binney, J. J. 2008, MNRAS, 390, 429

Morrison, H. L. 1993, AJ, 106, 578

Morrison, H. L., Flynn, C., \& Freeman, K. C. 1990, ApJ, 100, 1191

Myeong, G. C., Evans, N. W., Belokurov, V., Amorisco, N. C., \& Koposov, S. E. 2018a, MNRAS, 475, 1537

Myeong, G. C., Evans, N. W., Belokurov, V., Sanders, J. L., \& Koposov, S. E. 2018b, ApJ, 863, L28

Newberg, H. J., \& Carlin, J. L. 2016, in Tidal Streams in the Local Group and Beyond (Cham: Springer International Publishing), Astrophys. Space Sci. Lib., 420

Nissen, P. E., \& Schuster, W. J. 2010, A\&A, 511, L10

Odenkirchen, M., Grebel, E. K., Rockosi, C. M., et al. 2001, ApJ, 548, L165

Perryman, M. A. C., Lindegren, L., Kovalevsky, J., et al. 1997, A\&A, 323, 49

Pillepich, A., Vogelsberger, M., Deason, A., et al. 2014, MNRAS, 444, 237

Posti, L., Helmi, A., Veljanoski, J., \& Breddels, M. A. 2018, A\&A, 615, A70
Price-Whelan, A. M., \& Bonaca, A. 2018, ApJ, 863, L20

Re Fiorentin, P., Helmi, A., Lattanzi, M. G., \& Spagna, A. 2005, A\&A, 439, 551 Rockosi, C. M., Odenkirchen, M., Grebel, E. K., et al. 2002, AJ, 124, 349

Roederer, I. U., Sneden, C., Thompson, I. B., Preston, G. W., \& Shectman, S. A. 2010, ApJ, 711, 573

Sartoretti, P., Katz, D., Cropper, M., et al. 2018, A\&A, 616, A6

Schönrich, R., Binney, J., \& Dehnen, W. 2010, MNRAS, 403, 1829

Shipp, N., Drlica-Wagner, A., Balbinot, E., et al. 2018, AJ, 862, 114

Smith, M. C., Evans, N. W., Belokurov, V., et al. 2009, MNRAS, 399, 1223

Sohn, S. T., Watkins, L. L., Fardal, M. A., et al. 2018, AJ, 862, 52

Springel, V., \& White, S. D. M. 1999, MNRAS, 307, 162

Springel, V., White, S. D. M., Jenkins, A., et al. 2005, Nature, 435, 629

Taylor, M. B. 2005, in Astronomical Data Analysis Software And Systems XIV, eds. M. C. Britton, P. L. Shopbell, \& R. Ebert, 347

Taylor, M. B. 2006, in Astronomical Data Analysis Software and Systems XV, eds. D. Ponz, C. Gabriel, C. Arviset, \& E. Solano, 351

Tolstoy, E., Hill, V., \& Tosi, M. 2009, ARA\&A, 371, 371

Vandenberg, D. A., Brogaard, K., Leaman, R., \& Casagrande, L. 2013, ApJ, 775, 134

Van Der Walt, S. 2011, Comput. Sci. Eng., 13, 22

Weiler, M. 2018, A\&A, 617, A138

Williams, M. E. K., Steinmetz, M., Sharma, S., et al. 2011, ApJ, 728, 14

Wilson, J. C., Hearty, F., Skrutskie, M. F., et al. 2010, in Ground-based and Airborne Instrumentation for Astronomy III, Proc. SPIE, 7735, 77351C 


\section{Appendix A: Colour colour selection}

On the basis of the colour-colour diagram shown in Fig. A.1 we select possible sources that are heavily affected by extinction. The full sample of members is fit with a second-degree polynomial (dashed line). As explained Sect. 4.4, we consider as outliers those stars with a $\left(G-G_{\mathrm{BP}}\right)$ offset greater than 0.017 from the sequence (i.e. $5 \times$ the mean error in the colours used). The dashed line shown in the figure is offset by this 0.017 in $\left(G-G_{\mathrm{BP}}\right)$ to illustrate clearly which stars are considered to be reddened. All sources above the dashed line are marked as reddened sources.

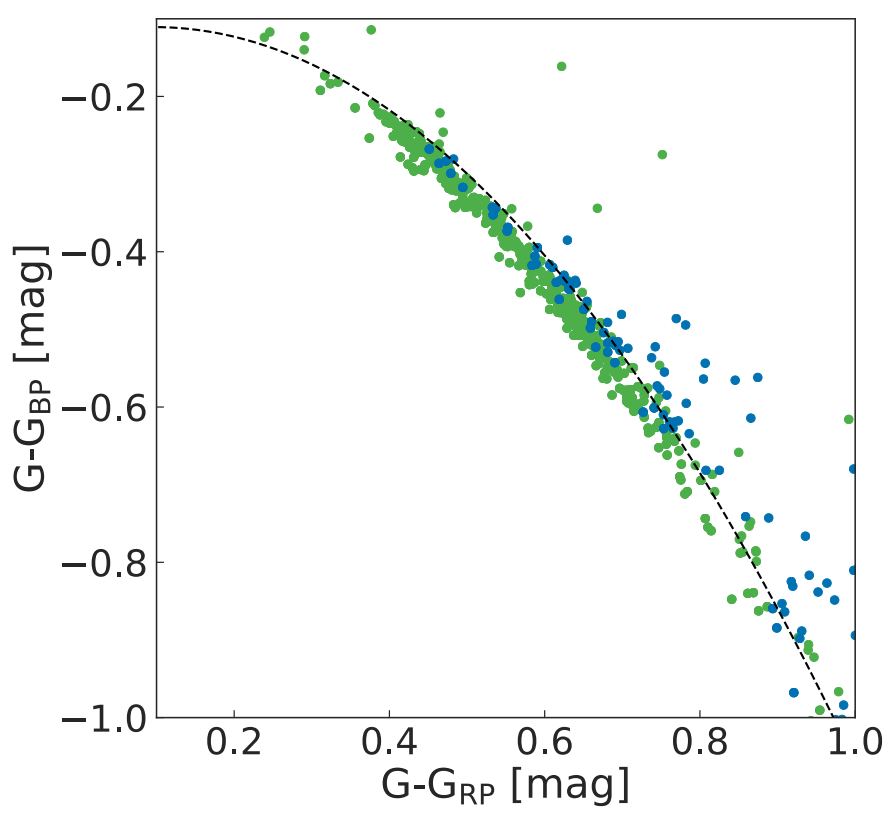

Fig. A.1. Colour-colour diagram used to identify sources that are likely affected by extinction. Sources that offset from the main population by more than $5 \times$ the mean error in the used colours, i.e. 0.017 in $\left(G-G_{\mathrm{BP}}\right)$, are considered to be reddened. The main population is fit with a seconddegree polynomial (dashed line). The dashed line is offset by 0.017 in $\left(G-G_{\mathrm{BP}}\right)$ to indicate which sources are reddened, i.e. those above the line. The members identified in the full 6D sample are shown with green markers, those without radial velocities are blue. 


\section{Appendix B: Table of members selection B}

Table B.1. Gaia DR2 source_ids for selection B.

\begin{tabular}{|c|c|c|c|c|}
\hline IDs members 1-105 & IDs members 106-210 & IDs members 211-315 & IDs members 316-419 & IDs members $420-523$ \\
\hline 4641558272441088 & 15542185269834624 & 18721418846252288 & 25052342374772736 & 40341188999965056 \\
\hline 44825306655035520 & 47948469433052800 & 48191667661477120 & 66174455213158656 & 88903903177506304 \\
\hline 109717108535569920 & 114370963299054720 & 129029789761784320 & 133659416611738496 & 148992625953529216 \\
\hline 166864465909649024 & 208313266841386496 & 214416419665198976 & 277505056837614336 & 297601582475179520 \\
\hline 298510126972435200 & 300034840362494592 & 317928636190603136 & 328660281995935232 & 334897296063939712 \\
\hline 338420371837484160 & 362606329112459136 & 365903386527108864 & 371779211025724032 & 378336075603390208 \\
\hline 414268497862196224 & 424315422799326464 & 565085174940343296 & 571826104634521856 & 579282859350500480 \\
\hline 579472799984173824 & 580075916471255040 & 597915355193425152 & 601485847406210688 & 604095572614068352 \\
\hline 609634774756212480 & 613717261429518336 & 618362698056754816 & 623835615967986560 & 640225833940128256 \\
\hline 641011469357926144 & 641984640227836288 & 642298482077861888 & 650878108749480960 & 658200993629082240 \\
\hline 660857241923470336 & 666167814367063296 & 675824721913920768 & 676184743253306752 & 690133560079137920 \\
\hline 694677944716195712 & 695472307507752320 & 703875080308465536 & 711475458731171712 & 715176071273608704 \\
\hline 720354564881582080 & 721179847141106816 & 722255002009942528 & 722917384750970752 & 724057994920894592 \\
\hline 724067027236448128 & 731949048138844800 & 735896535401139072 & 777558680244658176 & 780787744032780544 \\
\hline 793157490364148224 & 795010747276301952 & 809190053525078272 & 8094884334 & 80994 \\
\hline 812131247128723712 & 814140020513681024 & 821455518049745280 & 838151499037299328 & 839367249661110912 \\
\hline 839618793008582272 & 840616123070439552 & 841170654888167808 & 842363247047642368 & 845266198261944960 \\
\hline 846326471068781824 & 94885493888 & & 57744 & 877174369298101504 \\
\hline 877928256318192512 & 878629435499205632 & 879194790634452352 & 879287699366827520 & 885358240501948032 \\
\hline 892436552763913344 & 894379527249498496 & 900194908672475648 & & 9092555759755533920 \\
\hline 924073900341372160 & 926019211289351424 & 941117327005728000 & 951217299082189696 & 961149020114863872 \\
\hline 975473938637155968 & 1011601034570885504 & 1021904837208334848 & 1022757474116502400 & 1022993078842418432 \\
\hline 1033400437435614976 & 1049376272667191552 & 1143069060084735232 & 1155172720304980224 & 1160604689999075456 \\
\hline 1176187720407158912 & 1179353424836990720 & 1190457117888556160 & 1211628386079715840 & 1243915819907387392 \\
\hline 1244298759191228800 & 1255012675370113536 & 1258514207587827840 & 1260211058971256320 & 1261193202028905600 \\
\hline 1262839514533098368 & 1263921674493183488 & 1264302346034442496 & 1275876252107941888 & 1278333454435460096 \\
\hline 1278999758481584768 & 1286475922158536064 & 1288312793771274880 & 1317046296776785920 & 1319060876956649216 \\
\hline 1325856580370512768 & 1329198236725539456 & 1351435584519064576 & 1358807878703209472 & 1359836093873456768 \\
\hline 1361390494077900672 & 1375199947805963520 & 1375468537880511488 & 1376687518318241536 & 1390150935120171776 \\
\hline 1403917336097206144 & 1415635209471360256 & 1416077522383596160 & 1426065314211359488 & 1445550069004682368 \\
\hline 1454211574931259008 & 1454848260885706240 & 1459258161504411776 & 1461351734723634944 & 1465018949599024000 \\
\hline 1467009581041527168 & 1469066526780349312 & 1471944223587079808 & 147204376374 & 1475499116478182400 \\
\hline 1476416280975128832 & 1479656885339494656 & 1481262412833531520 & 1484142927140574976 & 1485907299704041088 \\
\hline 1498477363310839168 & 1500489435229902976 & 1501129694594779776 & 1501277853786344704 & 1512783143459215744 \\
\hline 1516361572771506176 & 1516500901510616960 & 1522392875086096384 & 1527475951701753984 & 1529110474518977920 \\
\hline 1532216221205478272 & 1533286423976243584 & 1534269318651955072 & 1543128667952320384 & 1543778891645673216 \\
\hline 1544941144151976832 & 1550080089702483584 & 1559682880662202240 & 1561486148450638080 & 1576029045153304448 \\
\hline 1590912446864098688 & 1595706764237530624 & 1601706279498493184 & 1601734905456540160 & 1603695231610033024 \\
\hline 1606083435288423680 & 1609793702916552448 & 1616751038836648192 & 1618407796700425856 & 1621470761217916800 \\
\hline 1639946061258413312 & 1651212997426399872 & 1659006954318690688 & 1661132860050868096 & 1680352357664352384 \\
\hline 1687099201530228352 & 1688495581297486336 & 1740372429681699712 & 1741837288407560192 & 1746823363186399104 \\
\hline 1770226575557939840 & 1785179585801889792 & 1880722993023978240 & 1897394608662403 & 1909569058536197760 \\
\hline 1915730687339037056 & 1920542906137425664 & 1927742920592283264 & 1959250663239949568 & 1969372801649974656 \\
\hline 2075971480449027840 & 2081319509311902336 & 2090990366909021312 & 2107177716389487744 & 2113756098755468288 \\
\hline 2118428129820961152 & 2119154219811372928 & 2143783830030463744 & 2152492992912794112 & 2223355928911050752 \\
\hline 2268048503896398720 & 2290361477475441664 & 2322233192826733184 & 2344587054493724288 & 2355425387285206016 \\
\hline 2373208853993082112 & 2416023871138662784 & 2417033428971348608 & 2436947439975372672 & 2438115774159097856 \\
\hline 2447968154259005952 & 2463622347979629952 & 2484686482506940416 & 2492679893385490944 & 2493751577920673664 \\
\hline 2497639347957300096 & 2515939172813427840 & 2518385517465704960 & 2519551171589880192 & 2533982360488142336 \\
\hline 2548084666562636544 & 2549249942728874368 & 2556488440091507584 & 2562681954730905344 & 2564879259999378560 \\
\hline 2565036284003715712 & 2565305629992853376 & 2568434290329701888 & 2577125551790493824 & 2577340815551111680 \\
\hline 2577877377225342464 & 2585460292309803264 & 2587112141027257472 & 2593008821887179008 & 2593099428517326848 \\
\hline 2597454555419529728 & 2604228169817599104 & 2610361657994140032 & 2652715636170048384 & 2658069914200047872 \\
\hline 2670534149811033088 & 2681582248805460864 & 2685833132557398656 & 2702017668840521088 & 2710316816966218496 \\
\hline 2715380235515703168 & 2716342071966879744 & 2731449911488518144 & 2747904614098575488 & 2753048786625056640 \\
\hline
\end{tabular}


H. H. Koppelman et al.: Characterization and history of the Helmi streams with Gaia DR2

Table B.1. continued.

\begin{tabular}{|c|c|c|c|c|}
\hline IDs members $1-105$ & IDs members 106-210 & IDs members 211-315 & IDs members 316-419 & IDs members 420-523 \\
\hline 2753174783785251328 & 2762507266682585728 & 2763829601214919040 & 2777125789169639296 & 2777896134503915648 \\
\hline 2782868607121209344 & 2807804328248244224 & 2816029465498234368 & 2838661293152878976 & 2841291982098062208 \\
\hline 2865194471533729152 & 2865540508457717376 & 2866048379751327616 & 2869555134648788736 & 2876439211309388672 \\
\hline 2882203332298969728 & 2890470899529272832 & 2891152566675457280 & 2901753198796969216 & 2902505745786910080 \\
\hline 2954185571135157504 & 2959451922593403904 & 2964823797107665664 & 3074369755487517184 & 3085891537839264896 \\
\hline 3085891537839267328 & 3124268582457078400 & 3140633331268375296 & 3165682645690499840 & 3179127443812906880 \\
\hline 3191801342547132544 & 3202308378739431936 & 3214420461393486208 & 3233974932096638592 & 3236724913756362624 \\
\hline 3244220864341745920 & 3246220914649655552 & 3258304272560352128 & 3267948604442696448 & 3270247545819617792 \\
\hline 3275579971053642624 & 3283448591659397504 & 3302997083768142464 & 3306026508883214080 & 3311451812788556800 \\
\hline 3318466112860232832 & 3344421699741253376 & 3360259919923274496 & 3368025671769397120 & 3368344461421835776 \\
\hline 3375760083236221568 & 3435000842026369280 & 3458106907086594304 & 3500260464905928832 & 3515304597176877056 \\
\hline 3520836313191203584 & 3612787302391075968 & 3638008690382625408 & 3638308577883569024 & 3642005964905520384 \\
\hline 3645723619877700352 & 3649703023041037696 & 3665517234359034752 & 3667980999398592384 & 3685879227632832640 \\
\hline 3693613948337587456 & 3694894948103300608 & 3696531536801618560 & 3697949460124343296 & 3697998658974002944 \\
\hline 3698500933925098496 & 3700419135039744896 & 3703984129693916928 & 3705595086027166976 & 3710778767956154112 \\
\hline 3712313170791794944 & 3712995761354141184 & 3716710186510880256 & 3718269255344140288 & 3719989475645411712 \\
\hline 3726338021425401728 & 3734341989334114304 & 3736775002407500416 & 3738979076544369920 & 3742101345970116224 \\
\hline 3742977210060826496 & 3767256797623849984 & 3773582902198012800 & 3784512979787195008 & 3786952418131994880 \\
\hline 3798431869281771264 & 3799979225739262592 & 3803865655746018176 & 3805053368822108160 & 3808640525507460864 \\
\hline 3812217030674464512 & 3812973391595125888 & 3815223026745362176 & 3817071305791426560 & 3830089282946013952 \\
\hline 3838162172195153280 & 3842793349531132928 & 3845801334171290240 & 3868120909114479104 & 3875684174723979904 \\
\hline 3887002066384290048 & 3892735332329204224 & 3892848582026608256 & 3894054127806485248 & 3897623005111511936 \\
\hline 3902635335025097344 & 3916126823734155264 & 3921020612550678784 & 3922882360614215808 & 3925374438078436736 \\
\hline 3927614040185794816 & 3928019553818870528 & 3928225540448909568 & 3929521177462206208 & 3932171034845348864 \\
\hline 3933991448143753344 & 3935464175249715328 & 3937879149461334784 & 3939125274092506880 & 3950135439936325376 \\
\hline 3959443664858300160 & 3960200884772486912 & 3961677219651037568 & 3965991806357554304 & 3966187038390853376 \\
\hline 3971115633621943424 & 3986709182404800640 & 3988327319923520384 & 3997068162486541056 & 4001150546081363584 \\
\hline 4008001534314802432 & 4018583985839228032 & 4024489531511197440 & 4027749961445908992 & 4317205919454821504 \\
\hline 4349990744807366144 & 4418988157460172288 & 4422328435129459584 & 4423061744962215680 & 4440446153372208640 \\
\hline 4457430485583460352 & 4466157103214240256 & 4494936029800093312 & 4534526935261529216 & 4561199025759521920 \\
\hline 4564066449004092928 & 4573372234384968192 & 4607911055710222848 & 4669519677214249856 & 4670120873852223488 \\
\hline 4679806226966950912 & 4692787168619498496 & 4731023544469431680 & 4738898315466921216 & 4754904073734461952 \\
\hline 4768015406298936960 & 4790679540699217152 & 4913172141123403648 & 4919544021460799232 & 4928561700436553856 \\
\hline 4963591625501132288 & 4965093901982556416 & 4987794109812160384 & 4988194744360498816 & 4998741805354135552 \\
\hline 5017192164520193536 & 5032050552340352384 & 5048696058874427008 & 5049085217270417152 & 5067943490953707008 \\
\hline 5079910055818560640 & 5122647149372975360 & 5155440732210734080 & 5167090027842913792 & 5187537405066478720 \\
\hline 5195968563309851136 & 5204327871039930752 & 5280057494615723648 & 5283335207500744832 & 5284231275125726336 \\
\hline 5296305287178801280 & 5340742599399608832 & 5360274938102023680 & 5366111940399568640 & 5388612346343578112 \\
\hline 5392578250427878912 & 5414925927344979328 & 5446633059547018496 & 5532006972050819584 & 5539345181373346560 \\
\hline 5552366427000089600 & 5558256888748624256 & 5563244926326975872 & 5621154039103070592 & 5658669238397675904 \\
\hline 5723101272620828800 & 5753657422310407808 & 5806384261908917248 & 5808577237852977664 & 5810465477272078208 \\
\hline 5818849597035446528 & 5840981323002935168 & 5851142528480811648 & 5897030680576289792 & 5907435045555029120 \\
\hline 5916061474500671232 & 5926846098713325440 & 5927992614459539584 & 6050982889930146304 & 6094133670436009984 \\
\hline 6109742818544552960 & 6144536275589632128 & 6170808423037019904 & 6221846137890957312 & 6255192092182279808 \\
\hline 6258000485397994880 & 6322846447087671680 & 6330365869671479296 & 6330586119889886336 & 6336613092877645440 \\
\hline 6356861870813950592 & 6387975296804951936 & 6393027243496440448 & 6485587710031536896 & 6490161850203676416 \\
\hline 6492720894795089280 & 6499030270473444096 & 6545771884159036928 & 6558166197703420928 & 6583674450854788096 \\
\hline 6615661065172699776 & 6620904533047264768 & 6626664771385047168 & 6695586523901966336 & 6701970872532935552 \\
\hline 6713733658378414848 & 6778212250043037184 & 6780343958276205824 & 6793770167779622144 & 6857300327589197696 \\
\hline 6866850891748542336 & 6909135394530431104 & 6914409197757803008 & & \\
\hline
\end{tabular}

